\title{
COMBINING MAXIMAL REGULARITY AND ENERGY ESTIMATES FOR TIME DISCRETIZATIONS OF QUASILINEAR PARABOLIC EQUATIONS
}

\author{
GEORGIOS AKRIVIS, BUYANG LI, AND CHRISTIAN LUBICH
}

\begin{abstract}
We analyze fully implicit and linearly implicit backward difference formula (BDF) methods for quasilinear parabolic equations, without making any assumptions on the growth or decay of the coefficient functions. We combine maximal parabolic regularity and energy estimates to derive optimal-order error bounds for the time-discrete approximation to the solution and its gradient in the maximum norm and energy norm.
\end{abstract}

\section{INTRODUCTION}

In this paper we study the time discretization of quasilinear parabolic differential equations by backward difference formulas (BDF). In contrast to the existing literature, we allow for solution-dependent coefficients in the equation that degenerate as the argument grows to infinity or approaches a singular set. To deal with such problems, we need to control the maximum norm of the error and possibly also of its gradient. As we show in this paper, such maximum norm estimates for BDF time discretizations become available by combining two techniques:

- discrete maximal parabolic regularity, as studied in Kovács, Li \& Lubich [14] based on the characterization of maximal $L^{p}$-regularity by Weis 24] and a discrete operator-valued Fourier multiplier theorem by Blunck [7]; and

- energy estimates, which are familiar for implicit Euler time discretizations and have become feasible for higher-order BDF methods (up to order 5) by the Nevanlinna-Odeh multiplier technique [19] as used in Akrivis \& Lubich [4.

In Section 2 we formulate the parabolic initial and boundary value problem and its time discretization by fully implicit and linearly implicit BDF methods, and we state our main results. For problems on a bounded Lipschitz domain $\Omega$ in $\mathbb{R}^{2}$ or $\mathbb{R}^{3}$ with sufficiently regular solutions, we obtain optimal-order error bounds in the maximum and energy norms. For problems on bounded smooth domains in arbitrary space dimension we obtain optimal-order error bounds even in the $L^{\infty}\left(0, T ; W^{1, \infty}(\Omega)\right)$ and $L^{2}\left(0, T ; H^{2}(\Omega)\right)$ norms.

The proof of these results makes up the remainder of the paper. In Section 3 we formulate a common abstract framework for both results and give a continuoustime perturbation result after which we model the stability proof of BDF methods

Received by the editor January 26, 2016.

2010 Mathematics Subject Classification. Primary 65M12, 65M15; Secondary 65L06.

Key words and phrases. BDF methods, maximal regularity, energy technique, parabolic equations, stability, maximum norm estimates. 
in Section 4. This proof relies on time-discrete maximal regularity and energy estimates. In Section 5 we study existence and uniqueness of the numerical solutions, and in Section 6 we discuss the consistency error of the fully and linearly implicit BDF methods. In the short Section 7 we combine the obtained estimates to prove the error bounds of our main results. In the remaining Sections 8 and 9 , which use different techniques of analysis, it is verified that the concrete parabolic problem and its time discretization fit into our abstract framework. In particular, the uniform discrete maximal parabolic regularity of the BDF methods is shown in the required $L^{q}$ and $W^{-1, q}$ settings.

While we restrict our attention in this paper to semidiscretization in time of standard quasilinear parabolic equations by BDF methods, the combination of discrete maximal regularity and energy estimates to obtain stability and error bounds in the maximum norm is useful for a much wider range of problems: for other time discretizations, for full discretizations (in combination with the discrete maximal regularity of semidiscrete finite element methods; see Li [16] and Li \& Sun [17]), and also for other classes of nonlinear parabolic equations. Such extensions are left to future work. The present paper can thus be viewed as a proof of concept for this powerful approach.

\section{Problem formulation and statement of the main Results}

2.1. Initial and boundary value problem. For a bounded domain $\Omega \subset \mathbb{R}^{d}$, a positive $T$, and a given initial value $u_{0}$, we consider the following initial and boundary value problem for a quasilinear parabolic equation, with homogeneous Dirichlet boundary conditions,

$$
\begin{cases}\partial_{t} u=\nabla \cdot(a(u) \nabla u) & \text { in } \Omega \times[0, T], \\ u=0 & \text { on } \partial \Omega \times[0, T], \\ u(\cdot, 0)=u_{0} & \text { in } \Omega .\end{cases}
$$

We assume that $a$ is a positive smooth function on the real line, but impose otherwise no growth or decay conditions on $a$ (for example, we may have $a(u)=e^{u}$ ). By the maximum principle, the solution of the above problem is bounded, provided the initial data $u_{0}$ is bounded; note that then there exists a positive number $K$ (depending on $\left.\|u\|_{L^{\infty}\left(0, T ; L^{\infty}(\Omega)\right)}\right)$ such that $K^{-1} \leq a(u(x, t)) \leq K$, for all $x \in \bar{\Omega}$ and $0 \leq t \leq T$. However, boundedness of the numerical approximations is not obvious.

Remark 2.1 (direct extensions). Our techniques and results can be directly extended to the following cases:

- The function $a$ is continuously differentiable and positive only in an interval $I \subset \mathbb{R}$ that contains all exact solution values, $u(x, t) \in I$; in particular, singularities in $a$ are allowed.

- $a$ is a function of $x, t$ and $u: a=a(x, t, u)$.

- $a(u)$ is a positive definite symmetric $d \times d$ matrix.

- A semilinear term $f(x, t, u, \nabla u)$ with a smooth function $f$ is added to the right-hand side of the differential equation. No growth conditions on $f$ need to be imposed, but we assume smoothness of the exact solution.

Operator notation: We consider $A(w)$ defined by $A(w) u:=-\nabla \cdot(a(w) \nabla u)$ as a linear operator on $L^{q}(\Omega)$, self-adjoint on $L^{2}(\Omega)$. 


\subsection{Fully and linearly implicit BDF methods.}

2.2.1. Fully implicit methods. We let $t_{n}=n \tau, n=0, \ldots, N$, be a uniform partition of the interval $[0, T]$ with time step $\tau=T / N$, and consider general $k$-step backward difference formulas (BDF) for the discretization of (2.1):

$$
\frac{1}{\tau} \sum_{j=0}^{k} \delta_{j} u_{n-j}=-A\left(u_{n}\right) u_{n},
$$

for $n=k, \ldots, N$, where $u_{1}, \ldots, u_{k-1}$ are sufficiently accurate given starting approximations and the coefficients of the method are given by

$$
\delta(\zeta)=\sum_{j=0}^{k} \delta_{j} \zeta^{j}=\sum_{\ell=1}^{k} \frac{1}{\ell}(1-\zeta)^{\ell} .
$$

The method is known to have order $k$ and to be $\mathrm{A}(\alpha)$-stable with angle $\alpha=$ $90^{\circ}, 90^{\circ}, 86.03^{\circ}, 73.35^{\circ}, 51.84^{\circ}, 17.84^{\circ}$ for $k=1, \ldots, 6$, respectively; see [12, Section V.2]. $\mathrm{A}(\alpha)$-stability is equivalent to $|\arg \delta(\zeta)| \leq 180^{\circ}-\alpha$ for $|\zeta| \leq 1$. Note that the first- and second-order BDF methods are A-stable, that is, $\operatorname{Re} \delta(\zeta) \geq 0$ for $|\zeta| \leq 1$.

2.2.2. Linearly implicit methods. Since equation (2.2) is in general nonlinear in the unknown $u_{n}$, we will also consider the following linearly implicit modification:

$$
\frac{1}{\tau} \sum_{j=0}^{k} \delta_{j} u_{n-j}=-A\left(\sum_{j=0}^{k-1} \gamma_{j} u_{n-j-1}\right) u_{n},
$$

for $n=k, \ldots, N$, with the coefficients $\gamma_{0}, \ldots, \gamma_{k-1}$ given by

$$
\gamma(\zeta)=\frac{1}{\zeta}\left[1-(1-\zeta)^{k}\right]=\sum_{i=0}^{k-1} \gamma_{i} \zeta^{i} .
$$

Notice that now the unknown $u_{n}$ appears in (2.3) only linearly; therefore, to advance with (2.3) in time, we only need to solve, at each time level, just one linear equation, which reduces to a linear system if we discretize also in space.

2.3. Main results. In this paper we prove the following two results.

Theorem 2.1. Let $\Omega \subset \mathbb{R}^{d}, d=2,3$, be a bounded Lipschitz domain. If the solution $u$ of (2.1) is sufficiently regular and the starting approximations are sufficiently accurate, then there exist $\tau_{0}>0$ and $C<\infty$ such that for stepsizes $\tau \leq \tau_{0}$ and $N \tau \leq T$, the fully and linearly implicit BDF methods (2.2) and (2.3), respectively, of order $k \leq 5$, have unique numerical solutions $u_{n} \in C(\bar{\Omega}) \cap H_{0}^{1}(\Omega)$ with errors bounded by

$$
\begin{aligned}
\max _{k \leq n \leq N}\left\|u_{n}-u\left(t_{n}\right)\right\|_{L^{\infty}(\Omega)} & \leq C \tau^{k}, \\
\left(\tau \sum_{n=k}^{N}\left\|u_{n}-u\left(t_{n}\right)\right\|_{H^{1}(\Omega)}^{2}\right)^{1 / 2} & \leq C \tau^{k} .
\end{aligned}
$$

Theorem 2.2. Let the bounded domain $\Omega \subset \mathbb{R}^{d}$ be smooth, where $d \geq 1$. If the solution $u$ of (2.1) is sufficiently regular and the starting approximations are sufficiently accurate, then there exist $\tau_{0}>0$ and $C<\infty$ such that for stepsizes $\tau \leq \tau_{0}$ and $N \tau \leq T$, the fully and linearly implicit BDF methods (2.2) and (2.3), 
respectively, of order $k \leq 5$, have unique numerical solutions $u_{n} \in C^{1}(\bar{\Omega}) \cap H^{2}(\Omega) \cap$ $H_{0}^{1}(\Omega)$ with errors bounded by

$$
\begin{array}{r}
\max _{k \leq n \leq N}\left(\left\|u_{n}-u\left(t_{n}\right)\right\|_{L^{\infty}(\Omega)}+\left\|\nabla u_{n}-\nabla u\left(t_{n}\right)\right\|_{L^{\infty}(\Omega)}\right) \leq C \tau^{k}, \\
\left(\tau \sum_{n=k}^{N}\left\|u_{n}-u\left(t_{n}\right)\right\|_{H^{2}(\Omega)}^{2}\right)^{1 / 2} \leq C \tau^{k} .
\end{array}
$$

Let us first comment on the regularity requirements. With some $q>d$, we need to assume in Theorem 2.1 that

$$
u \in C^{k+1}\left([0, T] ; W^{-1, q}(\Omega)\right) \cap C^{k}\left([0, T] ; L^{q}(\Omega)\right) \cap C\left([0, T] ; W^{1, q}(\Omega)\right),
$$

and in Theorem 2.2 that

$$
u \in C^{k+1}\left([0, T] ; L^{q}(\Omega)\right) \cap C^{k}\left([0, T] ; W^{1, q}(\Omega)\right) \cap C\left([0, T] ; W^{2, q}(\Omega)\right) .
$$

The errors in the initial data $e_{n}=u_{n}-u\left(t_{n}\right)$, for $n=0, \ldots, k-1$, need to satisfy the following bounds: in Theorem 2.1,

$$
\left(\tau \sum_{n=1}^{k-1}\left\|\frac{e_{n}-e_{n-1}}{\tau}\right\|_{W^{-1, q}(\Omega)}^{p}\right)^{\frac{1}{p}}+\left(\tau \sum_{n=1}^{k-1}\left\|e_{n}\right\|_{W^{1, q}(\Omega)}^{p}\right)^{\frac{1}{p}} \leq C \tau^{k},
$$

for some $p$ such that $2 / p+d / q<1$, and similarly in Theorem 2.2 with

$$
\left(\tau \sum_{n=1}^{k-1}\left\|\frac{e_{n}-e_{n-1}}{\tau}\right\|_{L^{q}(\Omega)}^{p}\right)^{\frac{1}{p}}+\left(\tau \sum_{n=1}^{k-1}\left\|e_{n}\right\|_{W^{2, q}(\Omega)}^{p}\right)^{\frac{1}{p}} \leq C \tau^{k} .
$$

It can be shown that these bounds are satisfied when the starting values are obtained with an algebraically stable implicit Runge-Kutta method of stage order $k$, such as the $k$-stage Radau collocation method.

Error bounds for BDF time discretizations of quasilinear parabolic differential equations have previously been obtained by Zlámal [25], for $k \leq 2$, and by Akrivis \& Lubich [4] for $k \leq 5$, using energy estimates. Implicit-explicit multistep methods for a particular class of such equations have been analyzed by Akrivis, Crouzeix \& Makridakis 2 by spectral and Fourier techniques. In those papers it is, however, assumed that the operators $A(u)$ are uniformly elliptic for $u \in H_{0}^{1}(\Omega)$, which amounts to assuming that the coefficient function $a$ is bounded on all $\mathbb{R}$ and has a strictly positive lower bound on all $\mathbb{R}$. This is a restrictive assumption that is not satisfied in many applications.

This restriction can be overcome only by controlling the maximum norm of the numerical solution, which is a major contribution of the present paper. Since no maximum principle is available for the BDF methods of order higher than 1 , the boundedness of the numerical solution is not obvious. While there are some results on maximum norm error bounds for implicit Euler and Crank-Nicolson time discretizations of linear parabolic equations by Schatz, Thomée \& Wahlbin [21, we are not aware of any such results for quasilinear parabolic equations as studied here.

In our view, even more interesting than the above particular results is the novel technique by which they are proved: by combining discrete maximal regularity and energy estimates. The combination of these techniques will actually yield $O\left(\tau^{k}\right)$ error bounds in somewhat stronger norms than stated in Theorems 2.1 and 2.2 , Moreover, we provide a concise abstract framework in which the combination of 
maximal regularity and energy estimates can be done and which allows for a common proof for both Theorems 2.1 and 2.2, as well as for extensions to more general quasilinear parabolic problems than (2.1) and to full discretizations.

\section{Abstract framework and basic approach in CONTINUOUs time}

As a preparation for the proof of Theorems 2.1 and 2.2. it is helpful to illustrate the approach taken in this paper first in a time-continuous and more abstract setting, which in particular applies to $A(w) u=-\nabla \cdot(a(w) \nabla u)$ as considered above.

3.1. Abstract framework. We formulate an abstract setting that works with Hilbert spaces $V \subset H$ and Banach spaces $D \subset W \subset X$ as follows: Let $H$ be the basic Hilbert space, and let $V$ be another Hilbert space that is densely and continuously imbedded in $H$. Together with the dual spaces we then have the familiar Gelfand triple of Hilbert spaces $V \subset H=H^{\prime} \subset V^{\prime}$ with dense and continuous imbeddings, and such that the restriction to $V \times H$ of the duality pairing $\langle\cdot, \cdot\rangle$ between $V$ and $V^{\prime}$ and of the inner product $(\cdot, \cdot)$ on $H$ coincide. Further, let $X \subset V^{\prime}$ be a Banach space and let $D \subset W \subset X$ be further Banach spaces. We denote the corresponding norms by $\|\cdot\|_{H},\|\cdot\|_{V},\|\cdot\|_{X},\|\cdot\|_{W}$, and $\|\cdot\|_{D}$, respectively, and summarize the continuous imbeddings:

$\begin{array}{lllll}V & \subset & H & \subset & V^{\prime} \\ \cup & & \cup & & \cup \\ D & \subset & W & \subset & X\end{array}$

For the existence of the numerical solution of the fully implicit BDF method we will further require that $D$ is compactly imbedded in $W$.

We have primarily the following two situations in mind:

(P1) For a bounded Lipschitz domain $\Omega \subset \mathbb{R}^{d}$ (with $d \leq 3$ ) we consider the usual Hilbert spaces $H=L^{2}(\Omega)$ and $V=H_{0}^{1}(\Omega)$, and in addition the Banach spaces $X=W^{-1, q}(\Omega)$ for suitable $q>d, W=C^{\alpha}(\bar{\Omega})$ with a small $\alpha>0$, and $D=W^{1, q}(\Omega) \cap H_{0}^{1}(\Omega)$.

(P2) For a smooth bounded domain $\Omega \subset \mathbb{R}^{d}$ (with arbitrary dimension $d$ ) we consider again the Hilbert spaces $H=L^{2}(\Omega)$ and $V=H_{0}^{1}(\Omega)$, and the Banach spaces $X=L^{q}(\Omega)$ with $q>d, W=C^{1, \alpha}(\bar{\Omega})$ with a small $\alpha>0$, and $D=W^{2, q}(\Omega) \cap H_{0}^{1}(\Omega)$.

We will work with the following five conditions:

(i) (W-locally uniform maximal regularity) For $w \in W$, the linear operator $-A(w)$ is the generator of an analytic semigroup on $X$ with domain $D(A(w))=D$ independent of $w$, and has maximal $L^{p}$-regularity: for $1<p<\infty$ there exists a real $C_{p}(w)$ such that the solution of the inhomogeneous initial value problem

$$
\dot{u}(t)+A(w) u(t)=f(t) \quad(0<t \leq T), \quad u(0)=0,
$$

is bounded by

$$
\|\dot{u}\|_{L^{p}(0, T ; X)}+\|A(w) u\|_{L^{p}(0, T ; X)} \leq C_{p}(w)\|f\|_{L^{p}(0, T ; X)} \quad \forall f \in L^{p}(0, T ; X) .
$$

Moreover, the bound is uniform in bounded sets of $W$ : for every $R>0$,

$$
C_{p}(w) \leq C_{p, R} \quad \text { if }\|w\|_{W} \leq R .
$$

We further require that the graph norms $\|\cdot\|_{X}+\|A(w) \cdot\|_{X}$ are uniformly equivalent to the norm $\|\cdot\|_{D}$ for $\|w\|_{W} \leq R$. 
(ii) (Control of the $W$-norm by maximal regularity) For some $1<p<\infty$, we have a continuous imbedding $W^{1, p}(0, T ; X) \cap L^{p}(0, T ; D) \subset L^{\infty}(0, T ; W)$ : there is $C_{p}<\infty$ such that for all $u \in W^{1, p}(0, T ; X) \cap L^{p}(0, T ; D)$,

$$
\|u\|_{L^{\infty}(0, T ; W)} \leq C_{p}\left(\|\dot{u}\|_{L^{p}(0, T ; X)}+\|u\|_{L^{p}(0, T ; D)}\right) .
$$

(iii) (V-ellipticity) $A(w)$ extends by density to a bounded linear operator $A(w)$ : $V \rightarrow V^{\prime}$, and for all $w \in W$ with $W$-norm bounded by $R$, the operator $A(w)$ is uniformly $V$-elliptic:

$$
\alpha_{R}\|u\|_{V}^{2} \leq\langle u, A(w) u\rangle \leq M_{R}\|u\|_{V}^{2} \quad \forall u \in V
$$

with $\alpha_{R}>0$ and $M_{R}<\infty$, where $\langle\cdot, \cdot\rangle$ denotes the duality pairing between $V$ and $V^{\prime}$.

(iv) (Operators with different arguments: $X$-norm estimate) For every $\varepsilon>0$, there is $C_{\varepsilon, R}<\infty$ such that for all $v, w \in W$ that are bounded by $R$ in the $W$-norm, and for all $u \in D$,

$$
\|(A(v)-A(w)) u\|_{X} \leq\left(\varepsilon\|v-w\|_{W}+C_{\varepsilon, R}\|v-w\|_{H}\right)\|u\|_{D} .
$$

(v) (Operators with different arguments: $V^{\prime}$-norm estimate) For every $\varepsilon>0$, there is $C_{\varepsilon, R}<\infty$ such that for all $v, w \in W$ that are bounded by $R$ in the $W$-norm, and for all $u \in D$,

$$
\|(A(v)-A(w)) u\|_{V^{\prime}} \leq\left(\varepsilon\|v-w\|_{V}+C_{\varepsilon, R}\|v-w\|_{H}\right)\|u\|_{D} .
$$

Lemma 3.1. The operators given by $A(w) u=-\nabla \cdot(a(w) \nabla u)$ with homogeneous Dirichlet boundary conditions and a smooth positive function $a(\cdot)$ satisfy $(\mathrm{i})-(\mathrm{v})$ in the situations ( $\mathrm{P} 1)$ and $(\mathrm{P} 2)$ above.

The proof of this lemma will be given in Section 8 and Section 9 . In the cases (P1) and (P2) we actually have a stronger bound than (v):

$$
\|(A(v)-A(w)) u\|_{V^{\prime}} \leq C_{R}\|v-w\|_{H}\|u\|_{D} .
$$

3.2. A perturbation result. Suppose now that $u \in W^{1, p}(0, T ; X) \cap L^{p}(0, T ; D)$ solves

$$
\left\{\begin{array}{l}
\dot{u}(t)+A(u(t)) u(t)=f(t), \quad 0<t \leq T, \\
u(0)=u_{0}
\end{array}\right.
$$

and $u^{\star} \in W^{1, \infty}(0, T ; W) \cap L^{p}(0, T ; D)$ solves the perturbed equation

$$
\left\{\begin{array}{l}
\dot{u}^{\star}(t)+A\left(u^{\star}(t)\right) u^{\star}(t)=f(t)+d(t), \quad 0<t \leq T, \\
u^{\star}(0)=u_{0},
\end{array}\right.
$$

where the defect $d$ is bounded by

$$
\|d\|_{L^{p}(0, T ; X)} \leq \delta .
$$

As an illustration of the combined use of maximal $L^{p}$-regularity and energy estimates we prove the following result. Theorems 2.1 and 2.2 will be proved by transferring these arguments to the time-discrete setting. 
Proposition 3.1. In the above setting of (i)-(v) and (3.2)-(3.4) with $\delta>0$ sufficiently small, the error $e=u-u^{\star}$ between the solutions of (3.2) and (3.3) is bounded by

$$
\begin{aligned}
\|\dot{e}\|_{L^{p}(0, T ; X)}+\|e\|_{L^{p}(0, T ; D)} & \leq C \delta, \\
\|e\|_{L^{\infty}(0, T ; W)} & \leq C \delta,
\end{aligned}
$$

where $C$ depends on $\left\|u^{\star}\right\|_{W^{1, \infty}(0, T ; W)}$ and $\left\|u^{\star}\right\|_{L^{p}(0, T ; D)}$, but is independent of $\delta$.

Proof. (a) (Error equation) We rewrite the equation in (3.2) in the form

$$
\dot{u}(t)+A\left(u^{\star}(t)\right) u(t)=\left(A\left(u^{\star}(t)\right)-A(u(t))\right) u(t)+f(t)
$$

and see that the error $e=u-u^{\star}$ satisfies the error equation

$$
\dot{e}(t)+A\left(u^{\star}(t)\right) e(t)=\left(A\left(u^{\star}(t)\right)-A(u(t))\right) u(t)-d(t) .
$$

Obviously, $e(0)=0$. To simplify the notation, we denote $\bar{A}(t):=A\left(u^{\star}(t)\right)$, and rewrite the error equation with some arbitrary $\bar{t} \geq t$ as

$$
\dot{e}(t)+\bar{A}(\bar{t}) e(t)=(\bar{A}(\bar{t})-\bar{A}(t)) e(t)+\left(A\left(u^{\star}(t)\right)-A(u(t))\right) u(t)-d(t),
$$

i.e.,

$$
\dot{e}(t)+\bar{A}(\bar{t}) e(t)=\widehat{d}(t)
$$

with

$$
\widehat{d}(t):=(\bar{A}(\bar{t})-\bar{A}(t)) e(t)-\left(A\left(u^{\star}(t)\right)-A(u(t))\right) u(t)-d(t) .
$$

(b) (Maximal regularity) We denote

$$
R=\left\|u^{\star}\right\|_{L^{\infty}(0, T ; W)}+1 \quad \text { and } \quad B=\left\|u^{\star}\right\|_{L^{p}(0, T ; D)}+1
$$

and let $0<t^{*} \leq T$ be maximal such that

$$
\|u\|_{L^{\infty}\left(0, t^{*} ; W\right)} \leq R \quad \text { and } \quad\|u\|_{L^{p}\left(0, t^{*} ; D\right)} \leq B .
$$

By the maximal $L^{p}$-regularity (i) we immediately obtain from (3.7) for $\bar{t} \leq t^{*}$

$$
\|\dot{e}\|_{L^{p}(0, \bar{t} ; X)}+\|e\|_{L^{p}(0, \bar{t} ; D)} \leq C_{p, R}\|\widehat{d}\|_{L^{p}(0, \bar{t} ; X)} .
$$

By the bound (iv) and the assumed Lipschitz continuity of $u^{\star}:[0, T] \rightarrow W$, we have for any $\varepsilon>0$

$$
\begin{aligned}
\|\widehat{d}(t)\|_{X} & \leq C(\bar{t}-t)\|e(t)\|_{D} \\
& +\varepsilon\|e(t)\|_{W}\|u(t)\|_{D}+C_{\varepsilon, R}\|e(t)\|_{H}\|u(t)\|_{D}+\|d(t)\|_{X} .
\end{aligned}
$$

We take the second term on the left-hand side of (3.10) to power $p$ and denote it by

$$
\eta(t)=\|e\|_{L^{p}(0, t ; D)}^{p} .
$$

For the first term on the right-hand side of (3.11) we note that, by partial integration,

$$
\int_{0}^{\bar{t}}(\bar{t}-t)^{p}\|e(t)\|_{D}^{p} d t=p \int_{0}^{\bar{t}}(\bar{t}-t)^{p-1} \eta(t) d t .
$$


Hence we have from (3.10)

$$
\begin{aligned}
\eta(\bar{t}) \leq & C_{p, R}^{p}\|\widehat{d}\|_{L^{p}(0, \bar{t} ; X)}^{p} \\
\leq & C \int_{0}^{\bar{t}}(\bar{t}-t)^{p-1} \eta(t) d t \\
& +C\left(\varepsilon B\|e\|_{L^{\infty}(0, \bar{t} ; W)}+C_{\varepsilon, R} B\|e\|_{L^{\infty}(0, \bar{t} ; H)}+\|d\|_{L^{p}(0, \bar{t} ; X)}\right)^{p} .
\end{aligned}
$$

With a Gronwall inequality, we therefore obtain from (3.10)

$\|\dot{e}\|_{L^{p}(0, \bar{t} ; X)}+\|e\|_{L^{p}(0, \bar{t} ; D)} \leq C \varepsilon\|e\|_{L^{\infty}(0, \bar{t} ; W)}+C_{\varepsilon, R, B}\|e\|_{L^{\infty}(0, \bar{t} ; H)}+C\|d\|_{L^{p}(0, \bar{t} ; X)}$, and we note that by property (ii) the left-hand term dominates $\|e\|_{L^{\infty}(0, \bar{t} ; W)}$. For sufficiently small $\varepsilon$ we can therefore absorb the first term of the right-hand side in the left-hand side to obtain

$$
\|\dot{e}\|_{L^{p}\left(0, t^{*} ; X\right)}+\|e\|_{L^{p}\left(0, t^{*} ; D\right)} \leq C\|e\|_{L^{\infty}\left(0, t^{*} ; H\right)}+C \delta .
$$

(c) (Energy estimate) To bound the first term on the right-hand side of (3.12) we use the energy estimate obtained by testing (3.5) with $e$ :

$$
\frac{1}{2} \frac{d}{d t}\|e(t)\|_{H}^{2}+\left\langle e(t), A\left(u^{\star}(t)\right) e(t)\right\rangle=\left\langle e(t),\left(A\left(u^{\star}(t)\right)-A(u(t))\right) u(t)\right\rangle-\langle e(t), d(t)\rangle .
$$

The bound of (v) yields

$$
\begin{aligned}
\left\langle e,\left(A\left(u^{\star}\right)-A(u)\right) u\right\rangle & \leq\|e\|_{V}\left(\varepsilon\|e\|_{V}+C_{\varepsilon, R}\|e\|_{H}\right)\|u\|_{D} \\
& \leq\left(2 \varepsilon\|e\|_{V}^{2}+C_{\varepsilon, R}\|e\|_{H}^{2}\right)\|u\|_{D} .
\end{aligned}
$$

Integrating from 0 to $t \leq t^{*}$, using the $V$-ellipticity (iii) and absorbing the term with $\|e\|_{V}^{2}$, we therefore obtain

$$
\|e(t)\|_{H}^{2}+\int_{0}^{t}\|e(s)\|_{V}^{2} d s \leq C \int_{0}^{t}\|e(s)\|_{H}^{2} d s+C \int_{0}^{t}\|d(s)\|_{V^{\prime}}^{2} d s,
$$

and the Gronwall inequality then yields

$$
\|e(t)\|_{H} \leq C \delta, \quad 0 \leq t \leq t^{*} .
$$

(d) (Complete time interval) Inserting the previous bound in (3.12), we obtain

$$
\|\dot{e}\|_{L^{p}\left(0, t^{*} ; X\right)}+\|e\|_{L^{p}\left(0, t^{*} ; D\right)} \leq C \delta,
$$

which by (ii) further implies

$$
\|e\|_{L^{\infty}\left(0, t^{*} ; W\right)} \leq C \delta .
$$

Hence, for sufficiently small $\delta$ we have the strict inequalities

$$
\|u\|_{L^{\infty}\left(0, t^{*} ; W\right)}<R \text { and }\|u\|_{L^{p}\left(0, t^{*} ; D\right)}<B .
$$

In view of the maximality of $t^{*}$ with (3.9) this is possible only if $t^{*}=T$.

Remark 3.1. If condition (ii) is strengthened to

$$
\|u\|_{C^{\alpha}([0, T] ; W)} \leq C_{\alpha, p}\left(\|\dot{u}\|_{L^{p}(0, T ; X)}+\|u\|_{L^{p}(0, T ; D)}\right),
$$

with some $\alpha>0$, then the statement of Proposition 3.1 remains valid under the weaker condition $u, u^{\star} \in W^{1, p}(0, T ; X) \cap L^{p}(0, T ; D)$, which is symmetric in $u$ and $u^{\star}$. The proof remains essentially the same. 


\section{Stability estimate for BDF methods}

4.1. Abstract framework for the time discretization. We work again with the abstract framework (i)-(v) of the previous section and consider in addition the following property of the BDF time discretization. Here we denote for a sequence $\left(v_{n}\right)_{n=1}^{N}$ and a given stepsize $\tau$

$$
\left\|\left(v_{n}\right)_{n=1}^{N}\right\|_{L^{p}(X)}=\left(\tau \sum_{n=1}^{N}\left\|v_{n}\right\|_{X}^{p}\right)^{1 / p},
$$

which is the $L^{p}(0, N \tau ; X)$ norm of the piecewise constant function taking the values $v_{n}$. We will work with the following discrete analog of condition (i).

(i') (W-locally uniform discrete maximal regularity) For $w \in W$, the linear operator $-A(w)$ has discrete maximal $L^{p}$-regularity for the BDF method: for $1<p<\infty$ there exists a real $C_{p}(w)$ such that for every stepsize $\tau>0$ the numerical solution determined by

$$
\dot{u}_{n}+A(w) u_{n}=f_{n} \quad(k \leq n \leq N) \quad \text { with } \quad \dot{u}_{n}=\frac{1}{\tau} \sum_{j=0}^{k} \delta_{j} u_{n-j}
$$

for starting values $u_{0}=\cdots=u_{k-1}=0$, is bounded by

$$
\left\|\left(\dot{u}_{n}\right)_{n=k}^{N}\right\|_{L^{p}(X)}+\left\|\left(A(w) u_{n}\right)_{n=k}^{N}\right\|_{L^{p}(X)} \leq C_{p}(w)\left\|\left(f_{n}\right)_{n=k}^{N}\right\|_{L^{p}(X)},
$$

where the constant is independent of $N$ and $\tau$. Moreover, the bound is uniform in bounded sets of $W$ : for every $R>0$,

$$
C_{p}(w) \leq C_{p, R} \quad \text { if }\|w\|_{W} \leq R .
$$

Lemma 4.1. For the operators given by $A(w) u=-\nabla \cdot(a(w) \nabla u)$ with homogeneous Dirichlet boundary conditions and a smooth positive function $a(\cdot)$ and the BDF methods up to order $k \leq 6$, the uniform discrete maximal regularity property (i') is fulfilled in the situations (P1) and (P2) of the previous section.

The proof of this lemma will be given in Section 8.2. We note that for a fixed $w$, such a result was first proved in [14] for $X=L^{q}$. The main novelty of Lemma 4.1] is thus the case $X=W^{-1, q}$ and the local $W$-uniformity of the result.

For the BDF methods of orders $k=3,4,5$, we need a further condition that complements (v):

(v') For all $v, w \in W$ that are bounded by $R$ in the $W$-norm, and for all $u \in V$,

$$
\|(A(v)-A(w)) u\|_{V^{\prime}} \leq C_{R}\|v-w\|_{W}\|u\|_{V} .
$$

This condition is trivially satisfied in the situations $(\mathrm{P} 1)$ and $(\mathrm{P} 2)$.

4.2. Stability estimate. In the following, let $\hat{u}_{n}=u_{n}$ for the fully implicit BDF method and $\hat{u}_{n}=\sum_{j=0}^{k-1} \gamma_{j} u_{n-j-1}$ for the linearly implicit BDF method.

Suppose now that $u_{n}, u_{n}^{\star} \in D(n=0, \ldots, N)$ solve

$$
\dot{u}_{n}+A\left(\hat{u}_{n}\right) u_{n}=f_{n}, \quad k \leq n \leq N,
$$

and the perturbed equation

$$
\dot{u}_{n}^{\star}+A\left(\hat{u}_{n}^{\star}\right) u_{n}^{\star}=f_{n}+d_{n}, \quad k \leq n \leq N,
$$

respectively, where it is further assumed that

$$
\left\|u_{m}^{\star}-u_{n}^{\star}\right\|_{W} \leq L(m-n) \tau, \quad 0 \leq n \leq m \leq N,
$$


and the defect $\left(d_{n}\right)$ is bounded by

$$
\left\|\left(d_{n}\right)_{n=k}^{N}\right\|_{L^{p}(X)} \leq \delta
$$

and the errors of the starting values are bounded by

$$
\frac{1}{\tau}\left\|\left(u_{i}-u_{i}^{\star}\right)_{i=0}^{k-1}\right\|_{L^{p}(X)} \leq \delta .
$$

We then have the following time-discrete version of Proposition 3.1 .

Proposition 4.1. Consider time discretization by a fully implicit or linearly implicit BDF method of order $k \leq 5$. In the above setting of the $W$-locally uniform discrete maximal regularity (i') and (ii)-(v) (and additionally (v') if $k=3,4,5$ ) and (4.2) -(4.6), there exist $\delta_{0}>0$ and $\tau_{0}>0$ such that for $\delta \leq \delta_{0}$ and $\tau \leq \tau_{0}$, the errors $e_{n}=u_{n}-u_{n}^{\star}$ between the solutions of (4.2) and (4.3) are bounded by

$$
\begin{aligned}
\left\|\left(\dot{e}_{n}\right)_{n=k}^{N}\right\|_{L^{p}(X)}+\left\|\left(e_{n}\right)_{n=k}^{N}\right\|_{L^{p}(D)} & \leq C \delta, \\
\left\|\left(e_{n}\right)_{n=k}^{N}\right\|_{L^{\infty}(W)} & \leq C \delta,
\end{aligned}
$$

where $C$ depends on $\left\|\left(u_{n}^{\star}\right)_{n=0}^{N}\right\|_{L^{\infty}(W)}$ and $\left\|\left(u_{n}^{\star}\right)_{n=0}^{N}\right\|_{L^{p}(D)}$ and on $L$ of (4.4), but is independent of $\delta$ and of $N$ and $\tau$ with $N \tau \leq T$.

This stability result will be proved in this and the next section.

4.3. Auxiliary results by Dahlquist and Nevanlinna \& Odeh. We will prove Proposition 4.1 for the linearly implicit scheme similarly to the proof of Proposition 3.1. To be able to use energy estimates in the time-discrete setting of BDF methods, we need the following auxiliary results.

Lemma 4.2 (Dahlquist [9; see also [6] and [12, Section V.6]). Let $\delta(\zeta)=\delta_{k} \zeta^{k}+$ $\cdots+\delta_{0}$ and $\mu(\zeta)=\mu_{k} \zeta^{k}+\cdots+\mu_{0}$ be polynomials of degree at most $k$ (and at least one of them of degree $k$ ) that have no common divisor. Let $(\cdot, \cdot)$ be an inner product with associated norm $|\cdot|$. If

$$
\operatorname{Re} \frac{\delta(\zeta)}{\mu(\zeta)}>0 \quad \text { for }|\zeta|<1
$$

then there exists a positive definite symmetric matrix $G=\left(g_{i j}\right) \in \mathbb{R}^{k \times k}$ and real $\kappa_{0}, \ldots, \kappa_{k}$ such that for $v_{0}, \ldots, v_{k}$ in the real inner product space,

$$
\left(\sum_{i=0}^{k} \delta_{i} v_{k-i}, \sum_{j=0}^{k} \mu_{j} v_{k-j}\right)=\sum_{i, j=1}^{k} g_{i j}\left(v_{i}, v_{j}\right)-\sum_{i, j=1}^{k} g_{i j}\left(v_{i-1}, v_{j-1}\right)+\left|\sum_{i=0}^{k} \kappa_{i} v_{i}\right|^{2} .
$$

In combination with the preceding result for the multiplier $\mu(\zeta)=1-\theta_{k} \zeta$, the following property of BDF methods up to order 5 becomes important.

Lemma 4.3 (Nevanlinna \& Odeh [19]). For $k \leq 5$, there exists $0 \leq \theta_{k}<1$ such that for $\delta(\zeta)=\sum_{\ell=1}^{k} \frac{1}{\ell}(1-\zeta)^{\ell}$,

$$
\operatorname{Re} \frac{\delta(\zeta)}{1-\theta_{k} \zeta}>0 \text { for }|\zeta|<1
$$

The smallest possible values of $\theta_{k}$ are

$$
\theta_{1}=\theta_{2}=0, \theta_{3}=0.0836, \theta_{4}=0.2878, \theta_{5}=0.8160 \text {. }
$$


Precise expressions for the optimal multipliers for the BDF methods of orders 3 , 4 , and 5 are given by Akrivis \& Katsoprinakis [3.

An immediate consequence of Lemma 4.3 and Lemma 4.2 is the relation

$$
\left(\sum_{i=0}^{k} \delta_{i} v_{k-i}, v_{k}-\theta_{k} v_{k-1}\right) \geq \sum_{i, j=1}^{k} g_{i j}\left(v_{i}, v_{j}\right)-\sum_{i, j=1}^{k} g_{i j}\left(v_{i-1}, v_{j-1}\right)
$$

with a positive definite symmetric matrix $G=\left(g_{i j}\right) \in \mathbb{R}^{k \times k}$; it is this inequality that will play a crucial role in our energy estimates.

4.4. Proof of Proposition 4.1 for the linearly implicit BDF methods. We subdivide the proof into four parts (a) to (d) that are analogous to the corresponding parts in the proof of Proposition 3.1. Parts (a)-(c) apply to both the linearly and fully implicit BDF methods, whereas the argument in part (d) does not work for the fully implicit method.

(a) (Error equation) We rewrite the equation for $u_{n}$ in the form

$$
\dot{u}_{n}+A\left(\hat{u}_{n}^{\star}\right) u_{n}=\left(A\left(\hat{u}_{n}^{\star}\right)-A\left(\hat{u}_{n}\right)\right) u_{n}+f_{n}
$$

and see that the errors $e_{n}=u_{n}-u_{n}^{\star}$ satisfy the error equation, for $n \leq N$,

$$
\dot{e}_{n}+A\left(\hat{u}_{n}^{\star}\right) e_{n}=\left(A\left(\hat{u}_{n}^{\star}\right)-A\left(\hat{u}_{n}\right)\right) u_{n}-d_{n} .
$$

We abbreviate $A_{n}=A\left(\hat{u}_{n}^{\star}\right)$. For an arbitrary $m \leq N$ and for $k \leq n \leq m$ we further have the error equation with a fixed operator

$$
\dot{e}_{n}+A_{m} e_{n}=\widehat{d}_{n}:=\left(A_{m}-A_{n}\right) e_{n}+\left(A\left(\hat{u}_{n}^{\star}\right)-A\left(\hat{u}_{n}\right)\right) u_{n}-d_{n} .
$$

If we redefine $e_{0}=\cdots=e_{k-1}=0$, then there appear extra defects for $n=$ $k, \ldots, 2 k-1$, which are bounded by $\delta$ by condition (4.6) and are subsumed in $d_{n}$ in the following.

(b) (Maximal regularity) We denote

$$
R=\left\|\left(u_{n}^{\star}\right)_{n=0}^{N}\right\|_{L^{\infty}(W)}+1 \quad \text { and } \quad B=\left\|\left(u_{n}^{\star}\right)_{n=0}^{N}\right\|_{L^{p}(D)}+1,
$$

and let $M \leq N$ be maximal such that

$$
\left\|\left(u_{n}\right)_{n=0}^{M-1}\right\|_{L^{\infty}(W)} \leq R \quad \text { and } \quad\left\|\left(u_{n}\right)_{n=0}^{M-1}\right\|_{L^{p}(D)} \leq B .
$$

By the discrete maximal $L^{p}$-regularity (i') we obtain from (4.9) that

$$
\left\|\left(\dot{e}_{n}\right)_{n=k}^{m}\right\|_{L^{p}(X)}+\left\|\left(e_{n}\right)_{n=k}^{m}\right\|_{L^{p}(D)} \leq C_{p, R}\left\|\left(\widehat{d}_{n}\right)_{n=k}^{m}\right\|_{L^{p}(X)},
$$

and by the bounds (iv) and (4.4), we have, for any $\varepsilon>0$,

$$
\begin{aligned}
\left\|\widehat{d}_{n}\right\|_{X} \leq & \left\|d_{n}\right\|_{X}+C_{R} L(m-n) \tau\left\|e_{n}\right\|_{D} \\
& +\varepsilon\left\|e_{n}\right\|_{W}\left\|u_{n}\right\|_{D}+C_{\varepsilon, R}\left\|e_{n}\right\|_{H}\left\|u_{n}\right\|_{D} \quad \text { for } k \leq n \leq m \leq M .
\end{aligned}
$$

We take the second term on the left-hand side of (4.12) to power $p$ and denote it by

$$
\eta_{m}=\left\|\left(e_{n}\right)_{n=k}^{m}\right\|_{L^{p}(D)}^{p}
$$


For the second term on the right-hand side of (4.13) we note that, by partial summation,

$$
\begin{aligned}
\tau \sum_{n=k}^{m}(m-n)^{p} \tau^{p}\left\|e_{n}\right\|_{D}^{p} & =\tau \sum_{n=k}^{m-1}\left((m-n)^{p}-(m-n-1)^{p}\right) \tau^{p-1} \eta_{n} \\
& \leq C \tau \sum_{n=k}^{m}((m-n) \tau)^{p-1} \eta_{n} .
\end{aligned}
$$

Hence we have from (4.12)

$$
\begin{aligned}
\eta_{m} \leq & C_{p, R}^{p}\left\|\left(\widehat{d}_{n}\right)_{n=k}^{m}\right\|_{L^{p}(X)}^{p} \leq C \tau \sum_{n=k}^{m}((m-n) \tau)^{p-1} \eta_{n} \\
& +C\left(\varepsilon B\left\|\left(e_{n}\right)_{n=k}^{m}\right\|_{L^{\infty}(W)}+C B\left\|\left(e_{n}\right)_{n=k}^{m}\right\|_{L^{\infty}(H)}+\left\|\left(d_{n}\right)_{n=k}^{m}\right\|_{L^{p}(X)}\right)^{p} .
\end{aligned}
$$

With a discrete Gronwall inequality, we therefore obtain from (4.12) that

$$
\begin{aligned}
\left\|\left(\dot{e}_{n}\right)_{n=k}^{M}\right\|_{L^{p}(X)}+\left\|\left(e_{n}\right)_{n=k}^{M}\right\|_{L^{p}(D)} \leq C \varepsilon\left\|\left(e_{n}\right)_{n=k}^{M}\right\|_{L^{\infty}(W)} & \\
& +C\left\|\left(e_{n}\right)_{n=k}^{M}\right\|_{L^{\infty}(H)}+C\left\|\left(d_{n}\right)_{n=k}^{M}\right\|_{L^{p}(X)}
\end{aligned}
$$

Next we show that by property (ii) the left-hand side dominates $\left\|\left(e_{n}\right)_{n=k}^{M}\right\|_{L^{\infty}(W)}$. We write $\delta(\zeta)=(1-\zeta) \mu(\zeta)$, where the polynomial $\mu(\zeta)$ of degree $k-1$ has no zeros in the closed unit disk and, therefore,

$$
\frac{1}{\mu(\zeta)}=\sum_{n=0}^{\infty} \chi_{n} \zeta^{n}, \quad \text { where } \quad\left|\chi_{n}\right| \leq \rho^{n} \quad \text { with } \rho<1 .
$$

It follows that

$$
\frac{e_{n}-e_{n-1}}{\tau}=\sum_{j=0}^{n} \dot{e}_{n-j} \chi_{j}
$$

and

$$
\left\|\left(\frac{e_{n}-e_{n-1}}{\tau}\right)_{n=k}^{M}\right\|_{L^{p}(X)} \leq C\left\|\left(\dot{e}_{n}\right)_{n=k}^{M}\right\|_{L^{p}(X)} .
$$

If we denote by $e(t)$ the piecewise linear function that interpolates the values $e_{n}$, then we have

$$
\|\dot{e}\|_{L^{p}(0, M \tau ; X)}=\left\|\left(\frac{e_{n}-e_{n-1}}{\tau}\right)_{n=k}^{M}\right\|_{L^{p}(X)}
$$

and

$$
\|e\|_{L^{p}(0, M \tau ; D)} \leq C\left\|\left(e_{n}\right)_{n=k}^{M}\right\|_{L^{p}(D)} .
$$

Combining the above inequalities and using property (ii) for $e(t)$, we thus obtain

$$
\left\|\left(e_{n}\right)_{n=k}^{M}\right\|_{L^{\infty}(W)} \leq C\left(\left\|\left(\dot{e}_{n}\right)_{n=k}^{M}\right\|_{L^{p}(X)}+\left\|\left(e_{n}\right)_{n=k}^{M}\right\|_{L^{p}(D)}\right) .
$$

For sufficiently small $\varepsilon$ we can therefore absorb the first term of the right-hand side of (4.14) in the left-hand side to obtain

$$
\left\|\left(\dot{e}_{n}\right)_{n=k}^{M}\right\|_{L^{p}(X)}+\left\|\left(e_{n}\right)_{n=k}^{M}\right\|_{L^{p}(D)} \leq C\left\|\left(e_{n}\right)_{n=k}^{M}\right\|_{L^{\infty}(H)}+C \delta .
$$


(c) (Energy estimate) To bound the first term on the right-hand side of (4.15) we use the energy estimate obtained by testing (4.8) with $e_{n}-\theta_{k} e_{n-1}$ with $\theta_{k}$ from Lemma 4.3.

$$
\frac{1}{\tau}\left(e_{n}-\theta_{k} e_{n-1}, \sum_{j=0}^{k} \delta_{j} e_{n-j}\right)+\left\langle e_{n}, A_{n} e_{n}\right\rangle-\theta_{k}\left\langle e_{n-1}, A_{n} e_{n}\right\rangle=\left\langle e_{n}-\theta_{k} e_{n-1}, \widetilde{d}_{n}\right\rangle
$$

with

$$
\widetilde{d}_{n}=\left(A\left(\hat{u}_{n}^{\star}\right)-A\left(\hat{u}_{n}\right)\right) u_{n}-d_{n} .
$$

Now, with the notation $E_{n}:=\left(e_{n}, \ldots, e_{n-k+1}\right)^{T}$ and the norm $\left|E_{n}\right|_{G}$ given by

$$
\left|E_{n}\right|_{G}^{2}=\sum_{i, j=1}^{k} g_{i j}\left(e_{n-k+i}, e_{n-k+j}\right),
$$

using (4.7), we can estimate the first term on the left-hand side from below in the form

$$
\left(e_{n}-\theta_{k} e_{n-1}, \sum_{j=0}^{k} \delta_{j} e_{n-j}\right) \geq\left|E_{n}\right|_{G}^{2}-\left|E_{n-1}\right|_{G}^{2} .
$$

In the following we denote by $\|\cdot\|_{n}$ the norm given by $\|v\|_{n}^{2}=\left\langle v, A_{n} v\right\rangle$, which by (iii) is equivalent to the $V$-norm. Furthermore,

$$
\left\langle e_{n}-\theta_{k} e_{n-1}, A_{n} e_{n}\right\rangle=\left\|e_{n}\right\|_{n}^{2}-\theta_{k}\left\langle e_{n-1}, A_{n} e_{n}\right\rangle,
$$

whence, obviously,

$$
\left\langle e_{n}-\theta_{k} e_{n-1}, A_{n} e_{n}\right\rangle \geq\left(1-\frac{\theta_{k}}{2}\right)\left\|e_{n}\right\|_{n}^{2}-\frac{\theta_{k}}{2}\left\|e_{n-1}\right\|_{n}^{2} .
$$

Moreover,

$$
\left\langle e_{n}-\theta_{k} e_{n-1}, \widetilde{d}_{n}\right\rangle \leq \varepsilon\left(\left\|e_{n}\right\|_{n}^{2}+\theta_{k}^{2}\left\|e_{n-1}\right\|_{n-1}^{2}\right)+C_{\varepsilon}\left\|\widetilde{d}_{n}\right\|_{V^{\prime}}^{2},
$$

for any positive $\varepsilon$. At this point, we need to relate $\left\|e_{n-1}\right\|_{n}$ back to $\left\|e_{n-1}\right\|_{n-1}$. We have, by the bounds (v') and (4.4),

$$
\|v\|_{n}^{2}-\|v\|_{n-1}^{2}=\left\langle v, A_{n} v\right\rangle-\left\langle v, A_{n-1} v\right\rangle=\left\langle v,\left(A_{n}-A_{n-1}\right) v\right\rangle \leq C \tau\|v\|_{V}^{2},
$$

so that

$$
\left\|e_{n-1}\right\|_{n}^{2} \leq(1+C \tau)\left\|e_{n-1}\right\|_{n-1}^{2} .
$$

Summing in (4.16) from $n=k$ to $m \leq M$, we obtain

$$
\left|E_{m}\right|_{G}^{2}+\rho \tau \sum_{n=k}^{m}\left\|e_{n}\right\|_{n}^{2} \leq C_{\varepsilon} \tau \sum_{n=k}^{m}\left\|\widetilde{d}_{n}\right\|_{V^{\prime}}^{2},
$$

with $\rho:=1-\theta_{k}-\left(1+\theta_{k}\right) \varepsilon>0$.

To estimate $\widetilde{d}_{n}$, we note that the bound of ( $\mathrm{v}$ ) yields

$$
\begin{aligned}
\left\|\left(A\left(\hat{u}_{n}^{\star}\right)-A\left(\hat{u}_{n}\right)\right) u_{n}\right\|_{V^{\prime}} & \leq \sum_{j=0}^{k}\left(\varepsilon\left\|e_{n-j}\right\|_{V}+C_{\varepsilon, R}\left\|e_{n-j}\right\|_{H}\right)\left\|u_{n}\right\|_{D} \\
& \leq \sum_{j=0}^{k}\left(2 \varepsilon\left\|e_{n-j}\right\|_{V}^{2}+C\left\|e_{n-j}\right\|_{H}^{2}\right)\left\|u_{n}\right\|_{D} .
\end{aligned}
$$


Absorbing the terms with $\left\|e_{n-j}\right\|_{V}^{2}$ we therefore obtain, for $k \leq m \leq M$,

$$
\left\|e_{m}\right\|_{H}^{2}+\tau \sum_{n=k}^{m}\left\|e_{n}\right\|_{V}^{2} \leq C \tau \sum_{n=k}^{m}\left\|e_{n}\right\|_{H}^{2}+C \tau \sum_{n=k}^{m}\left\|d_{n}\right\|_{V^{\prime}}^{2},
$$

and the discrete Gronwall inequality then yields

$$
\left\|e_{n}\right\|_{H} \leq C \delta, \quad k \leq n \leq M .
$$

(d) (Complete time interval) Inserting the previous bound in (4.15), we obtain

$$
\left\|\left(\dot{e}_{n}\right)_{n=k}^{M}\right\|_{L^{p}(X)}+\left\|\left(e_{n}\right)_{n=k}^{M}\right\|_{L^{p}(D)} \leq C \delta
$$

which by (ii) and the argument in part (b) above further implies

$$
\left\|\left(e_{n}\right)_{n=k}^{M}\right\|_{L^{\infty}(W)} \leq C \delta .
$$

For the linearly implicit BDF method, this implies that $\hat{u}_{M+1}=\sum_{j=0}^{k-1} \gamma_{j} u_{M-j}$ is bounded by $\left\|\hat{u}_{M+1}\right\|_{W} \leq C R$, and hence the above arguments can be repeated to yield that for sufficiently small $\delta$ the bounds (4.11) are also satisfied for $M+1$, which contradicts the maximality of $M$ unless $M=N$.

While parts (a)-(c) of the above proof apply also to the fully implicit BDF methods, the argument in part (d) does not work for the fully implicit method. Here we need some a priori estimate from the existence proof, which is established in the next section.

\section{Existence OF NUMERICAL SOlUtions FOR THE FULly IMPLICIT SCHEME}

While existence and uniqueness of the numerical solution are obvious for the linearly implicit BDF method (2.3), this is not so for the fully implicit method. In this section, we prove existence and uniqueness of the numerical solution for the fully implicit BDF method (2.2) and complete the proof of Proposition 4.1 for this method.

5.1. Schaefer's fixed point theorem. Existence of the solution of the fully implicit BDF method (2.2) is proved with the following result.

Lemma 5.1 (Schaefer's fixed point theorem [11, Chapter 9.2, Theorem 4]). Let W be a Banach space and let $\mathcal{M}: W \rightarrow W$ be a continuous and compact map. If the set

$$
\{\phi \in W: \phi=\theta \mathcal{M}(\phi) \text { for some } \theta \in[0,1]\}
$$

is bounded in $W$, then the map $\mathcal{M}$ has a fixed point.

5.2. Proof of the existence of the numerical solution and of Proposition 4.1 for fully implicit BDF methods. In the situation of Proposition 4.1. we assume that $u_{n} \in D, n=k, \ldots, M-1$, are solutions of (2.2) and satisfy

$$
\left\|\left(u_{n}\right)_{n=0}^{M-1}\right\|_{L^{\infty}(W)} \leq R
$$

with $R$ of (4.10), and prove existence of a numerical solution $u_{M}$ for (2.2), which also satisfies $\left\|u_{M}\right\|_{W} \leq R$.

We define a map $\mathcal{M}: W \rightarrow W$ in the following way. For any $\phi \in W$ we define

$$
\rho_{\phi}:=\min \left(\frac{\sqrt{\delta}}{\|\phi\|_{W}}, 1\right) .
$$


Clearly, $\rho_{\phi}$ depends continuously on $\phi \in W$, and

$$
\left\|\rho_{\phi} \phi\right\|_{W} \leq \sqrt{\delta}
$$

Then we define $e_{M}=\mathcal{M}(\phi)$ as the solution of the linear equation

$$
\begin{aligned}
\frac{1}{\tau} \sum_{j=0}^{k} \delta_{j} e_{M-j}= & A\left(u_{M}^{\star}\right) e_{M}+\left(A\left(u_{M}^{\star}+\rho_{\phi} \phi\right)-A\left(u_{M}^{\star}\right)\right) e_{M} \\
& +\left(A\left(u_{M}^{\star}+\rho_{\phi} \phi\right)-A\left(u_{M}^{\star}\right)\right) u_{M}^{\star}-d_{M} .
\end{aligned}
$$

Using the compact imbedding of $D$ in $W$, the fact that the resolvent operator $\left(\delta_{0} / \tau+A\left(u_{M}^{\star}+\rho_{\phi} \phi\right)\right)^{-1}$ maps from $X$ to $D$, and condition (iv) (with $\varepsilon=1$ ), it is shown that the map $\mathcal{M}$ is continuous and compact. Moreover, if the map $\mathcal{M}$ has a fixed point $\phi$ with $\rho_{\phi}=1$, then $e_{M}=\mathcal{M}(\phi)$ is a solution of

$$
\begin{aligned}
\frac{1}{\tau} \sum_{j=0}^{k} \delta_{j} e_{M-j}= & A\left(u_{M}^{\star}\right) e_{M}+\left(A\left(u_{M}^{\star}+e_{M}\right)-A\left(u_{M}^{\star}\right)\right) e_{M} \\
& +\left(A\left(u_{M}^{\star}+e_{M}\right)-A\left(u_{M}^{\star}\right)\right) u_{M}^{\star}-d_{M},
\end{aligned}
$$

and $u_{M}:=u_{M}^{\star}+e_{M}$ is the solution of (2.2) with $n=M$.

To apply Schaefer's fixed point theorem, we assume that $\phi \in W$ and $\phi=\theta \mathcal{M}(\phi)$ for some $\theta \in[0,1]$. To prove existence of a fixed point for the map $\mathcal{M}$, we only need to prove that all such $\|\phi\|_{W}$ are uniformly bounded.

Let $e_{M}=\mathcal{M}(\phi)$. Then $\phi=\theta e_{M}$ and (5.5) implies that $e_{M}$ is the solution of

$$
\begin{aligned}
\frac{1}{\tau} \sum_{j=0}^{k} \delta_{j} e_{M-j}= & A\left(u_{M}^{\star}\right) e_{M}+\left(A\left(u_{M}^{\star}+\theta \rho_{\phi} e_{M}\right)-A\left(u_{M}^{\star}\right)\right) e_{M} \\
& +\left(A\left(u_{M}^{\star}+\theta \rho_{\phi} e_{M}\right)-A\left(u_{M}^{\star}\right)\right) u_{M}^{\star}-d_{M}
\end{aligned}
$$

and

$$
\begin{aligned}
\frac{1}{\tau} \sum_{j=0}^{k} \delta_{j} e_{n-j}= & A\left(u_{n}^{\star}\right) e_{n}+\left(A\left(u_{n}^{\star}+e_{n}\right)-A\left(u_{n}^{\star}\right)\right) e_{n} \\
& +\left(A\left(u_{n}^{\star}+e_{n}\right)-A\left(u_{n}^{\star}\right)\right) u_{n}^{\star}-d_{n}
\end{aligned}
$$

for $n=k, \ldots, M-1$, satisfying

$$
\left\|\theta \rho_{\phi} e_{M}\right\|_{W} \leq \sqrt{\delta}
$$

In the same way as in Section 4.4, we obtain

$$
\left\|\left(\dot{e}_{n}\right)_{n=k}^{M}\right\|_{L^{p}(X)}+\left\|\left(e_{n}\right)_{n=k}^{M}\right\|_{L^{p}(D)} \leq C \delta
$$

and

$$
\left\|\left(e_{n}\right)_{n=0}^{M}\right\|_{L^{\infty}(W)} \leq C \delta .
$$

Since $\phi=\theta e_{M}$ with $\theta \in[0,1]$, the last inequality implies uniform boundedness of $\|\phi\|_{W}$ with respect to $\theta \in[0,1]$, and this implies the existence of a fixed point $\phi$ for the map $\mathcal{M}$ by Lemma 5.1. Moreover, in view of (5.11) and since the fixed point $\phi$ satisfies $\phi=e_{M}$, for sufficiently small $\delta$ we have

$$
\|\phi\|_{W} \leq \sqrt{\delta}, \quad \text { and so } \quad \rho_{\phi}=1 .
$$


This proves the existence of a solution of (2.2) for sufficiently small $\delta$, and that the solution satisfies (5.10) and (5.11) and hence also $\left\|u_{M}\right\|_{W} \leq R$, which completes the proof of Proposition 4.1 for the fully implicit BDF methods.

5.3. Uniqueness of the numerical solution. Suppose that there are two numerical solutions $u_{n}, \widetilde{u}_{n} \in D$ of (2.2), both with $W$-norm bounded by $R$ as in the proof of existence. By induction, we assume unique numerical solutions $u_{j}$ with $W$-norm bounded by $R$ for $j<n$. The difference $e_{n}=u_{n}-\widetilde{u}_{n}$ then satisfies the equation

$$
\frac{\delta_{0}}{\tau} e_{n}+A\left(\widetilde{u}_{n}\right) e_{n}=\left(A\left(u_{n}\right)-A\left(\widetilde{u}_{n}\right)\right) u_{n} .
$$

We test this equation with $e_{n}$ and note that, with some $\alpha_{R}>0$ depending on $R$, we have, using conditions (iii) and (v),

$$
\frac{\delta_{0}}{\tau}\left\|e_{n}\right\|_{H}^{2}+\alpha_{R}\left\|e_{n}\right\|_{V}^{2} \leq\left\|e_{n}\right\|_{V}\left(\varepsilon\left\|e_{n}\right\|_{V}+C_{\varepsilon, R}\left\|e_{n}\right\|_{H}\right)\left\|u_{n}\right\|_{D},
$$

which implies that there exists $\tau_{R}>0$ such that for $\tau \leq \tau_{R}$, we have $e_{n}=0$. We have thus shown uniqueness of the numerical solution $u_{n} \in D$ with $\left\|u_{n}\right\|_{W} \leq R$ for stepsizes $\tau \leq \tau_{R}$.

\section{COnSistency ERror}

The order of both the $k$-step fully implicit BDF method, described by the coefficients $\delta_{0}, \ldots, \delta_{k}$ and 1 , and of the explicit $k$-step BDF method, that is, the method described by the coefficients $\delta_{0}, \ldots, \delta_{k}$ and $\gamma_{0}, \ldots, \gamma_{k-1}$, is $k$, i.e.,

$$
\sum_{i=0}^{k}(k-i)^{\ell} \delta_{i}=\ell k^{\ell-1}=\ell \sum_{i=0}^{k-1}(k-i-1)^{\ell-1} \gamma_{i}, \quad \ell=0,1, \ldots, k .
$$

The defects (consistency errors) $d^{n}$ and $\tilde{d}^{n}$ of the schemes (2.2) and (2.3) for the solution $u$ of (2.1), i.e., the amounts by which the exact solution misses satisfying (2.2) and (2.3), respectively, are given by

$$
d_{n}=\frac{1}{\tau} \sum_{j=0}^{k} \delta_{j} u\left(t_{n-j}\right)+A\left(u\left(t_{n}\right)\right) u\left(t_{n}\right)
$$

and

$$
\tilde{d}_{n}=\frac{1}{\tau} \sum_{j=0}^{k} \delta_{j} u\left(t_{n-j}\right)+A\left(\sum_{j=0}^{k-1} \gamma_{j} u\left(t_{n-j-1}\right)\right) u\left(t_{n}\right),
$$

$n=k, \ldots, N$, respectively.

Lemma 6.1. Under the regularity requirements (2.8) or (2.9), the defects $d_{n}$ and $\tilde{d}_{n}$ are bounded by

$$
\max _{k \leq n \leq N}\left\|d_{n}\right\|_{W^{-1, q}(\Omega)} \leq C \tau^{k}, \quad \max _{k \leq n \leq N}\left\|\tilde{d}_{n}\right\|_{W^{-1, q}(\Omega)} \leq C \tau^{k}
$$

in the case of (2.8), and by

$$
\max _{k \leq n \leq N}\left\|d_{n}\right\|_{L^{q}(\Omega)} \leq C \tau^{k}, \quad \max _{k \leq n \leq N}\left\|\tilde{d}_{n}\right\|_{L^{q}(\Omega)} \leq C \tau^{k}
$$

in the case of (2.9). 
Proof. Since the proofs for (6.4) and (6.5) are almost identical, we just present the proof of (6.5). We first focus on the implicit scheme (2.2). Using the differential equation in (2.1), we rewrite (6.2) in the form

$$
d_{n}=\frac{1}{\tau} \sum_{j=0}^{k} \delta_{j} u\left(t_{n-j}\right)-\partial_{t} u\left(t_{n}\right) .
$$

By Taylor expanding about $t_{n-k}$, we see that, due to the order conditions of the implicit BDF method, i.e., the first equality in (6.1), leading terms of order up to $k-1$ cancel, and we obtain

$$
\begin{aligned}
d_{n}=\frac{1}{k !}[ & \frac{1}{\tau} \sum_{j=0}^{k} \delta_{j} \int_{t_{n-k}}^{t_{n-j}}\left(t_{n-j}-s\right)^{k} u^{(k+1)}(s) d s \\
& \left.-k \int_{t_{n-k}}^{t_{n}}\left(t_{n}-s\right)^{k-1} u^{(k+1)}(s) d s\right] ;
\end{aligned}
$$

here, we used the notation $u^{(m)}:=\frac{\partial^{m} u}{\partial t^{m}}$. Taking the $L^{q}$ norm on both sides of (6.7), we obtain the desired optimal order consistency estimate (6.5) for the scheme (2.2).

Next, concerning the scheme (2.3), from (6.2) and (6.3) we immediately obtain the following relation between $\tilde{d}_{n}$ and $d_{n}$

$$
\tilde{d}_{n}=d_{n}+\left(A\left(u\left(t_{n}\right)\right)-A\left(\hat{u}\left(t_{n}\right)\right)\right) u\left(t_{n}\right)
$$

with

$$
\hat{u}\left(t_{n}\right):=\sum_{i=0}^{k-1} \gamma_{i} u\left(t_{n-i-1}\right) .
$$

By Taylor expanding about $t_{n-k}$, the leading terms of order up to $k-1$ cancel again, this time due to the second equality in (6.1), and we obtain

$$
\begin{aligned}
u\left(t_{n}\right)-\hat{u}\left(t_{n}\right)=\frac{1}{(k-1) !}[ & \int_{t_{n-k}}^{t_{n}}\left(t_{n}-s\right)^{k-1} u^{(k)}(s) d s \\
& \left.-\sum_{j=0}^{k-1} \gamma_{j} \int_{t_{n-k}}^{t_{n-j-1}}\left(t_{n-j-1}-s\right)^{k-1} u^{(k)}(s) d s\right],
\end{aligned}
$$

whence, taking the $W^{1, q}$ norm on both sides of this relation, we immediately infer that

$$
\left\|u\left(t_{n}\right)-\hat{u}\left(t_{n}\right)\right\|_{W^{1, q}(\Omega)} \leq C \tau^{k} .
$$

Now, $\left(A\left(u\left(t_{n}\right)\right)-A\left(\hat{u}\left(t_{n}\right)\right)\right) u\left(t_{n}\right)=\nabla \cdot\left(\left(a\left(u\left(t_{n}\right)\right)-a\left(\hat{u}\left(t_{n}\right)\right)\right) \nabla u\left(t_{n}\right)\right)$, whence

$$
\begin{aligned}
& \left\|\left(A\left(u\left(t_{n}\right)\right)-A\left(\hat{u}\left(t_{n}\right)\right)\right) u\left(t_{n}\right)\right\|_{L^{q}(\Omega)} \\
& =\left\|\nabla \cdot\left(\left(a\left(u\left(t_{n}\right)\right)-a\left(\hat{u}\left(t_{n}\right)\right)\right) \nabla u\left(t_{n}\right)\right)\right\|_{L^{q}(\Omega)} \\
& \leq C\left\|a\left(u\left(t_{n}\right)\right)-a\left(\hat{u}\left(t_{n}\right)\right)\right\|_{L^{\infty}(\Omega)}\left\|u\left(t_{n}\right)\right\|_{W^{2, q}(\Omega)} \\
& \quad+C\left\|a\left(u\left(t_{n}\right)\right)-a\left(\hat{u}\left(t_{n}\right)\right)\right\|_{W^{1, q}(\Omega)}\left\|u\left(t_{n}\right)\right\|_{W^{1, \infty}(\Omega)} \\
& \leq C\left\|u\left(t_{n}\right)-\hat{u}\left(t_{n}\right)\right\|_{W^{1, q}(\Omega)} ;
\end{aligned}
$$


therefore, in view of (6.10), we have

$$
\left\|\left(A\left(u\left(t_{n}\right)\right)-A\left(\hat{u}\left(t_{n}\right)\right)\right) u\left(t_{n}\right)\right\|_{L^{q}(\Omega)} \leq C \tau^{k} .
$$

Combining (6.11) and the bound for $d_{n}$, we obtain the desired optimal order consistency estimate (6.5) also for the scheme (2.3).

\section{Proof of Theorems 2.1 and 2.2}

The cases (P1) and (P2) of Section 3.1] correspond to the situation in Theorems 2.1 and 2.2. respectively. Lemma 3.1 ensures that problems (P1) and (P2) are of the type considered in the abstract framework of Section 3.1, and Lemma 4.1 ensures the required uniform discrete maximal regularity of the BDF methods. Lemma 6.1 yields that the situation of Section 4.2 holds with $u_{n}^{\star}=u\left(t_{n}\right)$ and $\delta \leq C \tau^{k}$. The error bounds of Theorems 2.1 and 2.2 then follow from Proposition 4.1

It remains to give the proofs of Lemmas 3.1 and 4.1 This is done in the final two sections.

\section{8. $W$-LOCALLY UNIFORM MAXIMAL REGULARITY}

8.1. Proof of (i) in Lemma 3.1. Let $\Omega \subset \mathbb{R}^{d}$ be a bounded Lipschitz domain and consider the following initial and boundary value problem for a linear parabolic equation, with a time-independent self-adjoint operator,

$$
\begin{cases}\partial_{t} u(x, t)-\nabla \cdot(b(x) \nabla u(x, t))=0 & \text { for }(x, t) \in \Omega \times \mathbb{R}_{+}, \\ u(x, t)=0 & \text { for }(t, x) \in \partial \Omega \times \mathbb{R}_{+}, \\ u(x, 0)=u_{0}(x) & \text { for } x \in \Omega,\end{cases}
$$

where the coefficient $b(x)$ satisfies

$$
K_{0}^{-1} \leq b(x) \leq K_{0} .
$$

We consider $W=C^{\alpha}(\bar{\Omega})$ and $W=C^{1, \alpha}(\bar{\Omega})$ in the settings (P1) and (P2), respectively. In this section, we combine results from the literature and prove $W$-locally uniform maximal parabolic regularity of (8.1), where the constant depends only on $K_{0}$ and $\|b\|_{W}$.

Let $\left\{E_{2}(t)\right\}_{t>0}$ denote the semigroup of operators on $L^{2}(\Omega)$, which maps $u_{0}$ to $u(\cdot, t)$, given by (8.1) and let $A_{2}$ denote the generator of this semigroup. Then $\left\{E_{2}(t)\right\}_{t>0}$ extends to a bounded analytic semigroup on $L^{2}(\Omega)$, in the sector $\Sigma_{\theta}=$ $\{z \in \mathbb{C}: z \neq 0,|\arg z|<\theta\}$, where $\theta$ can be arbitrarily close to $\pi / 2$ (see [10,20]), and the kernel $G(t, x, y)$ of the semigroup $\left\{E_{2}(t)\right\}_{t>0}$ has an analytic extension to the right half-plane, satisfying (see [10, p. 103])

$$
|G(z, x, y)| \leq C_{\theta}|z|^{-\frac{d}{2}} \mathrm{e}^{-\frac{|x-y|^{2}}{C_{\theta}|z|}}, \quad \forall z \in \Sigma_{\theta}, \forall x, y \in \Omega, \quad \forall \theta \in(0, \pi / 2),
$$

where the constant $C_{\theta}$ depends only on $K_{0}$ and $\theta$. In other words, the rotated operator $A=-e^{i \theta} A_{2}$ satisfies the condition of [15, Theorem 8.6], with $m=2$ and $g(s)=C_{\theta} e^{-s^{2} / C_{\theta}}$ (see also [15, Remark 8.23]). As a consequence of [15, Theorem 8.6], $E_{2}(t)$ extends to an analytic semigroup $E_{q}(t)$ on $L^{q}(\Omega), 1<q<\infty$, which is $R$-bounded in the sector $\Sigma_{\theta}$ for all $\theta \in(0, \pi / 2)$ and the $R$-bound depends only on $C_{\theta}$ and $q$. (We refer to [15] for a discussion of the notion of $R$-boundedness.) To summarize, we have the following lemma. 
Lemma 8.1 (Angle of $R$-boundedness of the semigroup). For any given $1<q<\infty$, the semigroup $\left\{E_{q}(t): L^{q}(\Omega) \rightarrow L^{q}(\Omega)\right\}_{t>0}$ defined by the parabolic problem (8.1) is $R$-bounded in the sector $\Sigma_{\theta}=\{z \in \mathbb{C}:|\arg (z)|<\theta\}$ for all $\theta \in(0, \pi / 2)$, and the $R$-bound depends only on $K_{0}, \theta$ and $q$.

According to Weis' characterization of maximal $L^{p}$-regularity [24, Theorem 4.2], Lemma 8.1 implies the following two results.

Lemma 8.2 (Angle of $R$-boundedness of the resolvent). Let $A_{q}$ be the generator of the semigroup $\left\{E_{q}(t)\right\}_{t>0}$ defined by the parabolic problem (8.1), where $1<q<\infty$. Then the set $\left\{\lambda\left(\lambda-A_{q}\right)^{-1}: \lambda \in \Sigma_{\theta}\right\}$ is $R$-bounded in the sector $\Sigma_{\theta}=\{z \in \mathbb{C}$ : $|\arg (z)|<\theta\}$ for all $\theta \in(0, \pi)$, and the $R$-bound depends only on $K_{0}, \theta$ and $q$.

Lemma 8.3 (Maximal $L^{p}$-regularity). Let $A_{q}$ be the generator of the semigroup $\left\{E_{q}(t)\right\}_{t>0}$ defined by the parabolic problem (8.1). Then the solution $u(t)$ of the parabolic initial value problem

$$
\left\{\begin{array}{l}
u^{\prime}(t)=A_{q} u(t)+f(t), \quad t>0, \\
u(0)=0,
\end{array}\right.
$$

belongs to $D\left(A_{q}\right)$ for almost all $t \in \mathbb{R}_{+}$, and

$$
\left\|u^{\prime}\right\|_{L^{p}\left(\mathbb{R}_{+} ; L^{q}(\Omega)\right)}+\left\|A_{q} u\right\|_{L^{p}\left(\mathbb{R}_{+} ; L^{q}(\Omega)\right)} \leq C_{p, q}\|f\|_{L^{p}\left(\mathbb{R}_{+} ; L^{q}(\Omega)\right)},
$$

for all $f \in L^{p}\left(\mathbb{R}_{+} ; L^{q}(\Omega)\right)$ and $1<p, q<\infty$, where $C_{p, q}$ depends only on $p, q$ and $K_{0}$.

If the domain $\Omega$ is smooth and $b \in C^{1, \alpha}(\bar{\Omega})$, then (see [8, Chapter 3, Theorems 6.3-6.4]) by elliptic regularity

$$
\|u\|_{W^{2, q}(\Omega)} \leq C_{q}\left\|A_{q} u\right\|_{L^{q}(\Omega)},
$$

where the constant $C_{q}$ depends only on $K_{0}, q, \alpha$ and $\|b\|_{C^{1, \alpha}(\bar{\Omega})}$. Hence, Lemma 8.3 implies (i) for the case (P2).

In the case (P1), we need to use the following elliptic regularity result.

Lemma 8.4. For any given bounded Lipschitz domain $\Omega \subset \mathbb{R}^{d}, d=2,3$, the solution of the elliptic boundary value problem

$$
\begin{cases}\nabla \cdot(b(x) \nabla u)=f & \text { in } \Omega, \\ u=0 & \text { on } \partial \Omega,\end{cases}
$$

satisfies

$$
\|u\|_{W^{1, q}(\Omega)} \leq C_{q}\|f\|_{W^{-1, q}(\Omega)}, \quad \forall q_{d}^{\prime}<q<q_{d},
$$

where $q_{d}>2$ is some constant which depends on the domain and $1 / q_{d}+1 / q_{d}^{\prime}=1$, and the constant $C_{q}$ depends on $q, K_{0}, \Omega, \alpha$ and $\|b\|_{C^{\alpha}(\bar{\Omega})}$. In particular, $q_{2}>4$ and $q_{3}>3$ for any given bounded Lipschitz domain $\Omega \subset \mathbb{R}^{d}, d=2,3$.

Lemma 8.4 was proved in [13. Theorem 0.5] for constant coefficient $b(x)$, and this proof can be extended to variable coefficient $b(x)$ by a standard perturbation argument. By using Lemmas 8.3 and 8.4 , we can also prove the following maximal $L^{p}$-regularity on $W^{-1, q}(\Omega)$, which implies (i) for the case (P1) (with $X=W^{-1, q}(\Omega)$ and $\left.d<q<q_{d}\right)$. 
Lemma 8.5. If the domain $\Omega$ is Lipschitz continuous and $b \in C^{\alpha}(\bar{\Omega})$ for some $\alpha \in(0,1)$, then the solution of (8.4) satisfies

$$
\left\|\partial_{t} u\right\|_{L^{p}\left(\mathbb{R}_{+} ; W^{-1, q}(\Omega)\right)}+\|u\|_{L^{p}\left(\mathbb{R}_{+} ; W^{1, q}(\Omega)\right)} \leq C_{p, q}\|f\|_{L^{p}\left(\mathbb{R}_{+} ; W^{-1, q}(\Omega)\right)},
$$

for all $f \in L^{p}\left(\mathbb{R}_{+} ; W^{-1, q}(\Omega)\right), 1<p<\infty$ and $q_{d}^{\prime}<q<q_{d}$; the constant $C_{p, q}$ depends only on $p, q, K_{0}, \Omega, \alpha$ and $\|b\|_{C^{\alpha}(\bar{\Omega})}$.

Proof. Since the solution of (8.4) is given by

$$
u(t)=\int_{0}^{t} E_{q}(t-s) f(s) \mathrm{d} s,
$$

Lemma 8.3 implies that the map from $f$ to $A_{q} u$ given by the formula

$$
A_{q} u(t)=\int_{0}^{t} A_{q} E_{q}(t-s) f(s) \mathrm{d} s
$$

is bounded in $L^{p}\left(0, T ; L^{q}(\Omega)\right)$. In other words, if we define

$$
w(t):=-\int_{0}^{t} A_{q} E_{q}(t-s)\left(-A_{q}\right)^{-1 / 2} f(s) \mathrm{d} s,
$$

then we have

$$
\|w\|_{L^{p}\left(0, T ; L^{q}(\Omega)\right)} \leq C_{p, q}\left\|\left(-A_{q}\right)^{-1 / 2} f\right\|_{L^{p}\left(0, T ; L^{q}(\Omega)\right)},
$$

where the fractional power operator $\left(-A_{q}\right)^{-1 / 2}$ is well defined (due to the selfadjointness and positivity of $-A_{q}$ ) and commutes with $A_{q}$. It is straightforward to check that

$$
\nabla u=\nabla\left(-A_{q}\right)^{-1 / 2} w
$$

Since the Riesz transform $\nabla\left(-A_{q}\right)^{-1 / 2}$ is bounded on $L^{q}(\Omega)$ for $q_{d}^{\prime}<q<q_{d}$ (see Appendix), it follows that $\|\nabla u\|_{L^{p}\left(0, T ; L^{q}(\Omega)\right)} \leq C_{p, q}\|w\|_{L^{p}\left(0, T ; L^{q}(\Omega)\right)}$; therefore, in view of (8.9), we have

$$
\|\nabla u\|_{L^{p}\left(0, T ; L^{q}(\Omega)\right)} \leq C_{p, q}\left\|\left(-A_{q}\right)^{-1 / 2} f\right\|_{L^{p}\left(0, T ; L^{q}(\Omega)\right)} .
$$

Moreover, since $\left(-A_{q}\right)^{-1 / 2} \nabla$. is the dual of the Riesz transform $\nabla\left(-A_{q}\right)^{-1 / 2}$, it is also bounded on $L^{q}(\Omega)$ for any $q_{d}^{\prime}<q<q_{d}$, and so we have

$$
\begin{aligned}
\left\|\left(-A_{q}\right)^{-1 / 2} f\right\|_{L^{p}\left(0, T ; L^{q}(\Omega)\right)} & =\left\|\left(-A_{q}\right)^{-1 / 2} \nabla \cdot \nabla \Delta^{-1} f\right\|_{L^{p}\left(0, T ; L^{q}(\Omega)\right)} \\
& \leq C_{q}\left\|\nabla \Delta^{-1} f\right\|_{L^{p}\left(0, T ; L^{q}(\Omega)\right)},
\end{aligned}
$$

whence

$$
\left\|\left(-A_{q}\right)^{-1 / 2} f\right\|_{L^{p}\left(0, T ; L^{q}(\Omega)\right)} \leq C_{p, q}\|f\|_{L^{p}\left(0, T ; W^{-1, q}(\Omega)\right)} .
$$

Estimates (8.10) and (8.11) yield (8.8).

8.2. Proof of Lemma 4.1. In this section, we consider the BDF time discretization of (8.4):

$$
\begin{aligned}
& \frac{1}{\tau} \sum_{j=0}^{k} \delta_{j} u_{n-j}=A_{q} u_{n}+f_{n}, \quad n \geq k, \\
& u_{0}=0 \text { and } u_{1}, \ldots, u_{k-1} \text { given (possibly nonzero). }
\end{aligned}
$$

In view of Lemma 8.2, we have the following result, which implies Lemma 4.1 for the case (P2). 
Proposition 8.1 ([14, Theorems 4.1-4.2 and Remark 4.3]). For $1 \leq k \leq 6$, the solution of (8.12) -8.13) satisfies

$$
\begin{aligned}
& \left\|\left(\dot{u}_{n}\right)_{n=k}^{N}\right\|_{L^{p}\left(L^{q}(\Omega)\right)}+\left\|\left(A_{q} u_{n}\right)_{n=k}^{N}\right\|_{L^{p}\left(L^{q}(\Omega)\right)} \\
& \leq C_{p, q}\left(\tau \sum_{n=1}^{k-1}\left\|\frac{u_{n}-u_{n-1}}{\tau}\right\|_{L^{q}(\Omega)}^{p}\right)^{\frac{1}{p}}+C_{p, q}\left(\tau \sum_{n=1}^{k-1}\left\|A_{q} u_{n}\right\|_{L^{q}(\Omega)}^{p}\right)^{\frac{1}{p}} \\
& \quad+C_{p, q}\left\|\left(f_{n}\right)_{n=k}^{N}\right\|_{L^{p}\left(L^{q}(\Omega)\right)},
\end{aligned}
$$

for $1<p, q<\infty$, where the constant $C_{p, q}$ depends only on $K_{0}$ and $q$, i.e., it is independent of $\tau, N$ and $b$.

By applying Proposition 8.1, we prove the following result, which implies Lemma 4.1 for the case (P1) (with $X=W^{-1, q}(\Omega)$ and $d<q<q_{d}$ ).

Proposition 8.2. Let $1 \leq k \leq 6$. If the domain $\Omega$ is Lipschitz continuous and the coefficient satisfies $b \in C^{\alpha}(\bar{\Omega})$ for some $\alpha \in(0,1)$, then the solution of (8.12)(8.13) satisfies

$$
\begin{aligned}
& \left\|\left(\dot{u}_{n}\right)_{n=k}^{N}\right\|_{L^{p}\left(W^{-1, q}(\Omega)\right)}+\left\|\left(u_{n}\right)_{n=k}^{N}\right\|_{L^{p}\left(W^{1, q}(\Omega)\right)} \\
& \leq C_{p, q}\left(\tau \sum_{n=1}^{k-1}\left\|\frac{u_{n}-u_{n-1}}{\tau}\right\|_{W^{-1, q}(\Omega)}^{p}\right)^{\frac{1}{p}}+C_{p, q}\left(\tau \sum_{n=1}^{k-1}\left\|u_{n}\right\|_{W^{1, q}(\Omega)}^{p}\right)^{\frac{1}{p}} \\
& \quad+C_{p, q}\left\|\left(f_{n}\right)_{n=k}^{N}\right\|_{L^{p}\left(W^{-1, q}(\Omega)\right)},
\end{aligned}
$$

for all $1<p<\infty$ and $q_{d}^{\prime}<q<q_{d}$, and the constant $C_{p, q}$ depends only on $K_{0}, q$, $\Omega, \alpha$ and $\|b\|_{C^{\alpha}(\bar{\Omega})}$ (independent of $\tau$ and $\left.N\right)$.

Proof. In view of [14, Remark 4.3], we only need to consider the case $u_{0}=\cdots=$ $u_{k-1}=0$. The proof is a time-discrete analogue of the proof of Lemma 8.5

We consider the expansion

$$
\left(\frac{\delta(\zeta)}{\tau}-A_{q}\right)^{-1}=\tau \sum_{n=0}^{\infty} E_{n} \zeta^{n}, \quad|\zeta|<1,
$$

which yields (see, e.g., [14, Section 7])

$$
u_{n}=\sum_{j=k}^{n} \tau E_{n-j} f_{j} .
$$

Proposition 8.1 implies that the map from $\left(f_{n}\right)_{n=k}^{N}$ to $\left(A_{q} u_{n}\right)_{n=k}^{N}$ given by the formula

$$
A_{q} u_{n}=\sum_{j=k}^{n} \tau A_{q} E_{n-j} f_{j}
$$

is bounded in $L^{p}\left(L^{q}(\Omega)\right)$. In other words, if we define $w_{n}:=\left(-A_{q}\right)^{1 / 2} u_{n}$, then

$$
w_{n}=-\sum_{j=k}^{n} \tau A_{q} E_{n-j}\left(-A_{q}\right)^{-1 / 2} f_{j},
$$

and we have

$$
\left\|\left(w_{n}\right)_{n=1}^{N}\right\|_{L^{p}\left(L^{q}(\Omega)\right)} \leq C_{p, q}\left\|\left(\left(-A_{q}\right)^{-1 / 2} f_{n}\right)_{n=1}^{N}\right\|_{L^{p}\left(L^{q}(\Omega)\right)},
$$


where the fractional power operator $\left(-A_{q}\right)^{-1 / 2}$ commutes with $A_{q}$. Obviously,

$$
\nabla u_{n}=\nabla\left(-A_{q}\right)^{-1 / 2} w_{n}
$$

Since the Riesz transform $\nabla\left(-A_{q}\right)^{-1 / 2}$ is bounded on $L^{q}(\Omega)$ for $q_{d}^{\prime}<q<q_{d}$ (see Appendix), it follows that $\left\|\left(\nabla u_{n}\right)_{n=1}^{N}\right\|_{L^{p}\left(L^{q}(\Omega)\right)} \leq C_{p, q}\left\|\left(w_{n}\right)_{n=1}^{N}\right\|_{L^{p}\left(L^{q}(\Omega)\right)}$; therefore, in view of (8.16), we have

$$
\left\|\left(\nabla u_{n}\right)_{n=1}^{N}\right\|_{L^{p}\left(L^{q}(\Omega)\right)} \leq C_{p, q}\left\|\left(\left(-A_{q}\right)^{-1 / 2} f_{n}\right)_{n=1}^{N}\right\|_{L^{p}\left(L^{q}(\Omega)\right)} .
$$

Moreover, since $\left(-A_{q}\right)^{-1 / 2} \nabla$. is the dual of the Riesz transform $\nabla\left(-A_{q}\right)^{-1 / 2}$, it is also bounded on $L^{q}(\Omega)$ for any $q_{d}^{\prime}<q<q_{d}$, and so we have

$$
\begin{aligned}
\left\|\left(\left(-A_{q}\right)^{-1 / 2} f_{n}\right)_{n=1}^{N}\right\|_{L^{p}\left(L^{q}(\Omega)\right)} & =\left\|\left(\left(-A_{q}\right)^{-1 / 2} \nabla \cdot \nabla \Delta^{-1} f_{n}\right)_{n=1}^{N}\right\|_{L^{p}\left(L^{q}(\Omega)\right)} \\
& \leq C_{q}\left\|\left(\nabla \Delta^{-1} f_{n}\right)_{n=1}^{N}\right\|_{L^{p}\left(L^{q}(\Omega)\right)},
\end{aligned}
$$

whence

$$
\left\|\left(\left(-A_{q}\right)^{-1 / 2} f_{n}\right)_{n=1}^{N}\right\|_{L^{p}\left(L^{q}(\Omega)\right)} \leq C_{p, q}\left\|\left(f_{n}\right)_{n=1}^{N}\right\|_{L^{p}\left(W^{-1, q}(\Omega)\right)} .
$$

Estimates (8.17) and (8.18) yield (8.15).

\section{Sobolev and Related inequalities: Proof of Lemma 3.1}

A Banach space $X$ is said to be imbedded into another Banach space $Y$, denoted by $X \hookrightarrow Y$, if:

(a) $u \in X \Longrightarrow u \in Y$;

(b) $\|u\|_{Y} \leq C\|u\|_{X}$ for all $u \in X$, where $C$ is a constant independent of $u$.

The space $X$ is said to be compactly imbedded into $Y$, denoted by $X \hookrightarrow \hookrightarrow Y$, if in addition to (a)-(b) the following condition is satisfied:

(c) bounded subsets of $X$ are precompact subsets of $Y$.

Lemma 9.1. Let $X, Y$ and $Z$ be Banach spaces such that $X$ is compactly imbedded into $Y$, and $Y$ is imbedded into $Z$, i.e.,

$$
X \hookrightarrow \hookrightarrow Y \hookrightarrow Z
$$

Then, for any $\varepsilon>0$, there holds

$$
\|u\|_{Y} \leq \varepsilon\|u\|_{X}+C_{\varepsilon}\|u\|_{Z} \quad \forall u \in X .
$$

Proof. This lemma is probably well known, but since we did not find a reference, we include the short proof.

Suppose, on the contrary, that there exists $\varepsilon$ such that the inequality above does not hold for all $u \in X$. Then, there exists a sequence $u_{n} \in X, n=1,2, \ldots$, such that

$$
\left\|u_{n}\right\|_{Y} \geq \varepsilon\left\|u_{n}\right\|_{X}+n\left\|u_{n}\right\|_{Z}
$$

By a normalization (dividing $u_{n}$ by a constant), we can assume that $\left\|u_{n}\right\|_{Y}=1$ for all $n \geq 1$. Hence, we have

$$
\left\|u_{n}\right\|_{X} \leq 1 / \varepsilon \text { and }\left\|u_{n}\right\|_{Z} \leq 1 / n .
$$

On one hand, since $X$ is compactly embedded into $Y$, the boundedness of $u_{n}$ in $X$ implies the existence of a subsequence $u_{n_{k}}, k=1,2, \ldots$, which converges in $Y$ to some element $u \in Y \hookrightarrow Z$. Hence,

$$
\|u\|_{Y}=\lim _{k \rightarrow \infty}\left\|u_{n_{k}}\right\|_{Y}=1
$$


On the other hand, $\left\|u_{n}\right\|_{Z} \leq 1 / n$ implies that $u_{n_{k}}$ converges to the zero element in $Z$, which means that

$$
u=0 .
$$

Clearly, (9.1) and (9.2) contradict each other.

Lemma 9.2 (Sobolev imbedding). For $s>0,1<p, q<\infty$ and $d \geq 1$, we have:

(1) $W^{s, q}(\Omega) \hookrightarrow \hookrightarrow C^{\alpha}(\bar{\Omega}) \hookrightarrow L^{\infty}(\Omega)$ for $\alpha \in(0, s-d / q)$ if $s q>d$,

(2) $W^{s, q}(\Omega) \hookrightarrow \hookrightarrow C^{1, \alpha}(\bar{\Omega})$ for $\alpha \in(0, s-1-d / q)$ if $(s-1) q>d$,

(3) $W^{s, p}(\mathbb{R} ; X) \hookrightarrow L^{\infty}(\mathbb{R} ; X)$ if $s p>1$, where $X=L^{q}(\Omega)$ or $X=W^{-1, q}(\Omega)$,

(4) $H^{1}(\Omega) \hookrightarrow \hookrightarrow L^{q_{0}}(\Omega)$ for all $1 \leq q_{0}<2 d /(d-2)$ when $d \geq 2$, and $q_{0}=\infty$ when $d=1$.

Remark 9.1. (1)-(2) of Lemma 9.2 are immediate consequences of [5, p. xviii, Sobolev imbedding (18)]; (3) is a simple vector extension of (1); (4) can be found in [11, p. 272, Theorem 1].

Proof of (ii) in Lemma 3.1. For any $1<p, q<\infty$ and $r_{1}, r_{2}, r \geq 0$ such that $(1-\theta) r_{1}+\theta r_{2}=r$ for $\theta \in(0,1)$, we denote by $B_{p}^{r, q}(\Omega):=\left(W^{r_{1}, q}(\Omega), W^{r_{2}, q}(\Omega)\right)_{\theta, p}$ the Besov space of order $r$ (a real interpolation space between two Sobolev spaces, see [23]). Then, via Sobolev embedding, we have

$$
\begin{aligned}
& W^{1, p}\left(0, T ; L^{q}(\Omega)\right) \cap L^{p}\left(0, T ; W^{2, q}(\Omega)\right) \\
& \hookrightarrow L^{\infty}\left(0, T ;\left(L^{q}(\Omega), W^{2, q}(\Omega)\right)_{1-1 / p, p}\right) \quad \text { see [18, Proposition 1.2.10] } \\
& \simeq L^{\infty}\left(0, T ; B_{p}^{2-2 / p, q}(\Omega)\right) \\
& \hookrightarrow L^{\infty}\left(0, T ; C^{1, \alpha}(\Omega)\right) \quad \text { according to the definition } \\
&
\end{aligned}
$$

Proof of (iii)-(v) in Lemma 3.1. We first consider the setting (P2). Property (iii) is standard textbook material. To prove (iv), we note

$$
(A(v)-A(w)) u=\nabla \cdot((a(v)-a(w)) \nabla u)=\nabla(a(v)-a(w)) \cdot \nabla u+(a(v)-a(w)) \Delta u
$$

and estimate as follows:

$$
\begin{aligned}
\|(A(v)-A(w)) u\|_{L^{q}(\Omega)} \leq & C_{R}\|v-w\|_{W^{1, \infty}(\Omega)}\|u\|_{W^{1, q}(\Omega)} \\
& +C_{R}\|v-w\|_{L^{\infty}(\Omega)}\|u\|_{W^{2, q}(\Omega)} .
\end{aligned}
$$

Since $C^{1, \alpha}(\bar{\Omega})$ is compactly imbedded into $W^{1, \infty}(\Omega)$ and $L^{\infty}(\Omega)$, Lemma 9.1 gives us the inequalities, for arbitrary $\varepsilon>0$,

$$
\begin{aligned}
\|v-w\|_{W^{1, \infty}(\Omega)} & \leq \varepsilon\|v-w\|_{C^{1, \alpha}(\bar{\Omega})}+C_{\varepsilon}\|v-w\|_{L^{2}(\Omega)}, \\
\|v-w\|_{L^{\infty}(\Omega)} & \leq \varepsilon\|v-w\|_{C^{1, \alpha}(\bar{\Omega})}+C_{\varepsilon}\|v-w\|_{L^{2}(\Omega)} .
\end{aligned}
$$

The inequality of $(\mathrm{v})$ follows by estimating

$$
\langle\varphi,(A(v)-A(w)) u\rangle \leq C_{R}\|\varphi\|_{H^{1}(\Omega)}\|v-w\|_{L^{\bar{q}}(\Omega)}\|u\|_{W^{1, q}(\Omega)},
$$

where $1 / \bar{q}+1 / q=1 / 2$. Note that for the considered $q>d$ we have $\bar{q}=q /(q / 2-1)<$ $d /(d / 2-1)$, and so $H^{1}(\Omega)$ is compactly imbedded into $L^{\bar{q}}(\Omega)$ (see (4) of Lemma 9.2 or [1, Theorem 6.3$])$, so that by Lemma 9.1

$$
\|v-w\|_{L^{\bar{q}}(\Omega)} \leq \varepsilon\|v-w\|_{H^{1}(\Omega)}+C_{\varepsilon}\|v-w\|_{L^{2}(\Omega)} .
$$


We now turn to the setting (P1). Property (iii) is the same as in (P2), and (v) has actually been shown above. Property (iv) follows from estimating

$$
\langle\varphi,(A(v)-A(w)) u\rangle \leq C_{R}\|\varphi\|_{W^{1, q^{\prime}}(\Omega)}\|v-w\|_{L^{\infty}(\Omega)}\|u\|_{W^{1, q}(\Omega)},
$$

where $1 / q+1 / q^{\prime}=1$, and from the bound

$$
\|v-w\|_{L^{\infty}(\Omega)} \leq \varepsilon\|v-w\|_{C^{\alpha}(\bar{\Omega})}+C_{\varepsilon}\|v-w\|_{L^{2}(\Omega)},
$$

which follows from Lemma 9.1

\section{Appendix A. Boundedness of the Riesz transform}

Let $A_{q}: D\left(A_{q}\right) \rightarrow L^{q}(\Omega)$ be defined by $A_{q} u=\nabla \cdot(b(x) \nabla u)$, where $D\left(A_{q}\right)=\left\{u \in W^{1, q}(\Omega): \nabla \cdot(b(x) \nabla u) \in L^{q}(\Omega)\right\} \quad$ and $\quad b \in C^{\alpha}(\bar{\Omega})$ satisfies (8.2).

Lemma A.1. The Riesz transform $\nabla\left(-A_{q}\right)^{-1 / 2}$ is bounded on $L^{q}(\Omega)$ for $q_{d}^{\prime}<q<$ $q_{d}$, i.e.,

$$
\left\|\nabla\left(-A_{q}\right)^{-1 / 2} u\right\|_{L^{q}(\Omega)} \leq C_{q}\|u\|_{L^{q}(\Omega)}, \quad \text { for } q_{d}^{\prime}<q<q_{d},
$$

where the constant $C_{q}$ depends on $q, \Omega, \alpha$ and $\|b\|_{C^{\alpha}(\bar{\Omega})}$.

Proof. It has been proved in [22, Theorem B] that the Riesz transform $\nabla\left(-A_{q}\right)^{-1 / 2}$ is bounded on $L^{q}(\Omega)$ if and only if every solution of the homogeneous equation

$$
\nabla \cdot(b(x) \nabla u)=0
$$

in $\Omega \cap B_{2 r}\left(x_{0}\right)$ such that $u=0$ on $\partial \Omega \cap B_{2 r}\left(x_{0}\right)$ (if it is not empty) satisfies the following local estimate:

$$
\left(\frac{1}{r^{d}} \int_{\Omega \cap B_{r}\left(x_{0}\right)}|\nabla u|^{q} \mathrm{~d} x\right)^{\frac{1}{q}} \leq C\left(\frac{1}{r^{d}} \int_{\Omega \cap B_{2 r}\left(x_{0}\right)}|\nabla u|^{2} \mathrm{~d} x\right)^{\frac{1}{2}},
$$

for all $x_{0} \in \Omega$ and $0<r<r_{0}$, where $r_{0}$ is any given small positive constant such that $\Omega \cap B_{2 r_{0}}\left(x_{0}\right)$ is the intersection of $B_{2 r_{0}}\left(x_{0}\right)$ with a Lipschitz graph. It remains to prove (A.2).

Let $\omega$ be a smooth cut-off function which equals zero outside $B_{2 r}:=B_{2 r}\left(x_{0}\right)$ and equals 1 on $B_{r}$. Extend $u$ to be zero on $B_{2 r} \backslash \Omega$ and denote by $u_{2 r}$ the average of $u$ over $B_{2 r}$. Then (A.1) implies

$$
\nabla \cdot\left(b \nabla\left(\omega\left(u-u_{2 r}\right)\right)\right)=\nabla \cdot\left(b\left(u-u_{2 r}\right) \nabla \omega\right)+b \nabla \omega \cdot \nabla\left(u-u_{2 r}\right) \quad \text { in } \Omega,
$$

and the $W^{1, q}$ estimate (Lemma 8.4) implies

$$
\begin{aligned}
\left\|\omega\left(u-u_{2 r}\right)\right\|_{W^{1, q}(\Omega)} & \leq C\left\|\left(u-u_{2 r}\right) \nabla \omega\right\|_{L^{q}(\Omega)}+C\|\nabla \omega \cdot \nabla u\|_{W^{-1, q}(\Omega)} \\
& \leq C\left\|\left(u-u_{2 r}\right) \nabla \omega\right\|_{L^{q}(\Omega)}+C\|\nabla \omega \cdot \nabla u\|_{L^{s}(\Omega)} \\
& =C\left\|\left(u-u_{2 r}\right) \nabla \omega\right\|_{L^{q}\left(B_{2 r}\right)}+C\|\nabla \omega \cdot \nabla u\|_{L^{s}\left(B_{2 r}\right)} \\
& \leq C r^{-1}\|\nabla u\|_{L^{s}\left(B_{2 r}\right)},
\end{aligned}
$$

where $s=q d /(q+d)<q$ satisfies $L^{s}(\Omega) \hookrightarrow W^{-1, q}(\Omega)$ and $W^{1, s}(\Omega) \hookrightarrow L^{q}(\Omega)$. The last inequality implies

$$
\|\nabla u\|_{L^{q}\left(\Omega \cap B_{r}\right)} \leq C r^{-1}\|\nabla u\|_{L^{s}\left(\Omega \cap B_{2 r}\right)} .
$$

If $s \leq 2$, then one can derive

$$
\|\nabla u\|_{L^{q}\left(\Omega \cap B_{r}\right)} \leq C r^{d / q-d / 2}\|\nabla u\|_{L^{2}\left(\Omega \cap B_{2 r}\right)}
$$


by once more using Hölder's inequality on the right-hand side. Otherwise, one only needs a finite number of iterations of (A.4) to reduce $s$ to be less than 2. This completes the proof of (A.2.

\section{ACKNOWLEDGMENT}

The research stay of Buyang Li at the University of Tübingen was funded by the Alexander von Humboldt Foundation.

\section{REFERENCES}

[1] R. A. Adams and J. J. F. Fournier, Sobolev Spaces, 2nd ed., Pure and Applied Mathematics (Amsterdam), vol. 140, Elsevier/Academic Press, Amsterdam, 2003. MR2424078

[2] G. Akrivis, M. Crouzeix, and C. Makridakis, Implicit-explicit multistep methods for quasilinear parabolic equations, Numer. Math. 82 (1999), no. 4, 521-541, DOI 10.1007/s002110050429. MR.1701828

[3] G. Akrivis and E. Katsoprinakis, Backward difference formulae: new multipliers and stability properties for parabolic equations, Math. Comp. 85 (2016), no. 301, 2195-2216, DOI 10.1090/mcom3055. MR3511279

[4] G. Akrivis and C. Lubich, Fully implicit, linearly implicit and implicit-explicit backward difference formulae for quasi-linear parabolic equations, Numer. Math. 131 (2015), no. 4, 713-735, DOI 10.1007/s00211-015-0702-0. MR3422451

[5] H. Amann, Linear and Quasilinear Parabolic Problems. Vol. I: Abstract Linear Theory, Monographs in Mathematics, vol. 89, Birkhäuser Boston, Inc., Boston, MA, 1995. MR.1345385

[6] C. Baiocchi and M. Crouzeix, On the equivalence of A-stability and G-stability, Appl. Numer. Math. 5 (1989), no. 1-2, 19-22, DOI 10.1016/0168-9274(89)90020-2. Recent theoretical results in numerical ordinary differential equations. MR.979543

[7] S. Blunck, Maximal regularity of discrete and continuous time evolution equations, Studia Math. 146 (2001), no. 2, 157-176, DOI 10.4064/sm146-2-3. MR.1853519

[8] Y.-Z. Chen and L.-C. Wu, Second Order Elliptic Equations and Elliptic Systems, Translations of Mathematical Monographs, vol. 174, American Mathematical Society, Providence, RI, 1998. Translated from the 1991 Chinese original by Bei Hu. MR. 1616087

[9] G. Dahlquist, G-stability is equivalent to A-stability, BIT 18 (1978), no. 4, 384-401, DOI 10.1007/BF01932018. MR520750

[10] E. B. Davies, Heat Kernels and Spectral Theory, Cambridge Tracts in Mathematics, vol. 92, Cambridge University Press, Cambridge, 1989. MR990239

[11] L. C. Evans, Partial Differential Equations, 2nd ed., Graduate Studies in Mathematics, vol. 19, American Mathematical Society, Providence, RI, 2010. MR2597943

[12] E. Hairer and G. Wanner, Solving Ordinary Differential Equations II: Stiff and DifferentialAlgebraic Problems, $2^{\text {nd }}$ revised ed., Springer-Verlag, Berlin, Heidelberg, Springer Series in Computational Mathematics v. 14, 2002 (see MR2657217).

[13] D. Jerison and C. E. Kenig, The inhomogeneous Dirichlet problem in Lipschitz domains, J. Funct. Anal. 130 (1995), no. 1, 161-219, DOI 10.1006/jfan.1995.1067. MR1331981

[14] B. Kovács, B. Li, and C. Lubich, A-stable time discretizations preserve maximal parabolic regularity, SIAM J. Numer. Anal. 54 (2016), no. 6, 3600-3624, DOI 10.1137/15M1040918. MR 3582825

[15] P. C. Kunstmann and L. Weis, Maximal $L_{p}$-regularity for parabolic equations, Fourier multiplier theorems and $H^{\infty}$-functional calculus, Functional analytic methods for evolution equations, Lecture Notes in Math., vol. 1855, Springer, Berlin, 2004, pp. 65-311, DOI 10.1007/9783-540-44653-8_2. MR2108959

[16] B. Li, Maximum-norm stability and maximal $L^{p}$ regularity of FEMs for parabolic equations with Lipschitz continuous coefficients, Numer. Math. 131 (2015), no. 3, 489-516, DOI 10.1007/s00211-015-0698-5. MR 3395142

[17] B. Li and W. Sun, Regularity of the diffusion-dispersion tensor and error analysis of Galerkin FEMs for a porous medium flow, SIAM J. Numer. Anal. 53 (2015), no. 3, 1418-1437, DOI 10.1137/140958803. MR 3355773 
[18] A. Lunardi, Analytic Semigroups and Optimal Regularity in Parabolic Problems, Progress in Nonlinear Differential Equations and their Applications, 16, Birkhäuser Verlag, Basel, 1995. MR.1329547

[19] O. Nevanlinna and F. Odeh, Multiplier techniques for linear multistep methods, Numer. Funct. Anal. Optim. 3 (1981), no. 4, 377-423, DOI 10.1080/01630568108816097. MR636736

[20] E.-M. Ouhabaz, Gaussian estimates and holomorphy of semigroups, Proc. Amer. Math. Soc. 123 (1995), no. 5, 1465-1474, DOI 10.2307/2161136. MR 1232142

[21] A. H. Schatz, V. C. Thomée, and L. B. Wahlbin, Maximum norm stability and error estimates in parabolic finite element equations, Comm. Pure Appl. Math. 33 (1980), no. 3, 265-304, DOI 10.1002/cpa.3160330305. MR562737

[22] Z. Shen, Bounds of Riesz transforms on $L^{p}$ spaces for second order elliptic operators (English, with English and French summaries), Ann. Inst. Fourier (Grenoble) 55 (2005), no. 1, 173-197. MR2141694

[23] L. Tartar, An Introduction to Sobolev Spaces and Interpolation Spaces, Lecture Notes of the Unione Matematica Italiana, vol. 3, Springer, Berlin; UMI, Bologna, 2007. MR2328004

[24] L. Weis, Operator-valued Fourier multiplier theorems and maximal $L_{p}$-regularity, Math. Ann. 319 (2001), no. 4, 735-758, DOI 10.1007/PL00004457. MR1825406

[25] M. Zlámal, Finite element methods for nonlinear parabolic equations, RAIRO Anal. Numér. 11 (1977), no. 1, 93-107, 113. MR0502073

Department of Computer Science \& Engineering, University of Ioannina, 45110 IoanNINA, GreEce

E-mail address: akrivis@cse.uoi.gr

Department of Applied Mathematics, The Hong Kong Polytechnic University, Hung Hom, Hong KONG

E-mail address: buyang.li@polyu.edu.hk

Mathematisches Institut, Universität Tübingen, Auf Der Morgenstelle, D-72076 TüBingen, Germany

E-mail address: lubich@na.uni-tuebingen.de 